\title{
Interfacial diffusion in high-temperature deformation of composites: a discrete dislocation plasticity investigation
}

\author{
Siamak S. Shishvan \\ Department of Engineering, University of Cambridge, Trumpington Street, Cambridge CB2 1PZ, UK \\ Faculty of Civil Engineering, University of Tabriz, P.O. Box 51666-16471, Tabriz, Iran \\ Tresa M. Pollock \\ Materials Department, University of California Santa Barbara, CA 93106-5050, USA \\ Robert M. McMeeking \\ Department of Materials and Department of Mechanical Engineering, University of California Santa Barbara, CA 93106, USA \\ School of Engineering, University of Aberdeen, King's College, Aberdeen AB24 3UE, UK \\ Vikram S. Deshpande* \\ Department of Engineering, University of Cambridge, Trumpington Street, Cambridge CB2 1PZ, UK
}

\begin{abstract}
We present a discrete dislocation plasticity (DDP) framework to analyse the high temperature deformation of multiphase materials (composites) comprising a matrix and inclusions. Deformation of the phases is by climb-assisted glide of the dislocations while the particles can also deform due to stress-driven interfacial diffusion. The general framework is used to analyse the uniaxial tensile deformation of a composite comprising elastic particles with dislocation plasticity only present in the matrix phase. When dislocation motion is restricted to only glide within the matrix a strong size effect of the composite strength is predicted with the strength increasing with decreasing unit cell size due to dislocations forming pile-ups against the matrix/particle interface. Interfacial diffusion decreases the composite strength as it enhances the elongation of the elastic particles along the loading direction. When dislocation motion occurs by climb-assisted glide within the matrix the size effect of the strength is reduced as dislocations no longer arrange high energy pile-up structures but rather form lower energy dislocation cell networks. While interfacial diffusion again reduces the composite strength, in contrast to continuum plasticity predictions, the elongation of the particles is almost independent of the interfacial diffusion constant. Rather, in DDP the reduction in composite strength due to interfacial diffusion is a result of changes in the dislocation structures within the matrix and the associated enhanced dislocation climb rates in the matrix.
\end{abstract}

Keywords: High temperature composites, Discrete dislocation plasticity, Interfacial diffusion, Plastic deformation

\footnotetext{
${ }^{*}$ Corresponding author

Email address: vsd@eng. cam. ac.uk (Vikram S. Deshpande )
} 


\section{Introduction}

The creep strength of metal and inter-metallic matrix composite materials reinforced by elastic particles is of interest in high temperature, high strength components. The underlying principle is that strengthening develops from the constraint of the reinforcement on the matrix. Consequently, creep strength at high temperature will persist only if the constraint is maintained. Rosler et al. (1991) investigated this situation in the mechanical behaviour of $\gamma$-TiAl reinforced with $\mathrm{Ti}_{2} \mathrm{AlC}$ platelets in the temperature range $293 \mathrm{~K}$ to $1255 \mathrm{~K}$. At temperatures up to $1000 \mathrm{~K}$, the creep strength of the composite material at a given strain rate was found to be higher than that of the matrix alone. Such a temperature corresponds to half of the melting temperature of the matrix. As the temperature increases beyond $1000 \mathrm{~K}$, the relative composite strength decreases and at about $1250 \mathrm{~K}$ falls below that of the matrix. Typically, this loss of strength has been attributed to loss of constraint due to relative slip between the matrix and particle and/or mass transport from the sides to the top/bottom of long reinforcements. This provides additional strain of the composite material above and beyond that due to matrix flow. Such phenomena have been modelled employing continuum plasticity with the matrix considered as a power-law creeping solid; see for example Sofronis and McMeeking (1994) and more recently by Li et al. (2014). These continuum models are anticipated to be adequate if geometric length scales (e.g. the size of the reinforcements and their spacing) are much larger than the dislocation microstructure lengths such as dislocation cell sizes and mean dislocation travel distance.

Experimental studies on metal matrix composites (MMCs) at room temperature suggest that these continuum models are well suited for composites with inclusion sizes typically greater than $100 \mu \mathrm{m}$ but their applicability breaks down for composites with micron- or nano-sized inclusions; see Kamat et al. (1989). This so-called size effect is typically associated with dislocation micro-mechanisms in the metal matrix as predicted in the early pioneering work of Cleveringa et al. (1997) using discrete dislocation plasticity (DDP) with dislocation motion restricted to occur only by glide along specified slip planes. A number of high temperature MMCs such as single crystal superalloys consist of a ductile matrix (denoted $\gamma$ ) in which a large volume fraction of an ordered precipitation strengthening phase (denoted $\gamma^{\prime}$ ) resides. These $\gamma^{\prime}$ particles are typically no more than $400 \mathrm{~nm}$ in size and separated by matrix channels that could be as small as $10 \mathrm{~nm}$ wide. Continuum plasticity is expected to not provide an adequate description for the deformation of the matrix phase at high temperatures in such cases with DDP a more appropriate tool.

Superalloys and other high temperature MMCs are typically used at temperatures that considerably exceed $0.3 T_{\mathrm{m}}$, where $T_{\mathrm{m}}$ is the melting (liquidus) temperature of the alloy. At these elevated temperatures, the diffusion of vacancies and the associated climb of dislocations are expected to play a significant role. Discrete dislocation-based descriptions of plasticity at these high temperatures are in their infancy though there has been some recent notable progress. In particular, Zhu et al. (2012) developed phenomenological laws motivated by dislocation mechanics for the creep of nickel-based single crystal superalloys while Haghighat et al. (2013) included climb in discrete dislocation calculations to model creep of nickel superalloys without explicitly solving a boundary value problem of a unit cell of the superalloy. A numerical recipe to solve boundary value problems wherein plasticity occurs by the motion of discrete 
dislocations was proposed by Van der Giessen and Needleman (1995) in the context of glide motion of dislocations. It involves solving a many body interaction problem involving the discrete dislocations together with a complimentary more conventional elasticity boundary value problem. This framework has now been extended to include glide and climb of dislocations; see for example Ayas et al. (2014), Danas and Deshpande (2013) and Keralavarma and Benzerga (2015). Ayas et al. (2015) employed this framework to investigate the deformation of an MMC wherein the reinforcement phase comprises elastic inclusions and the matrix deforms by climb-assisted glide of the dislocations. This study demonstrated that dislocation climb results in the formation of low energy dislocation structures within the matrix and a reduction in the sensitivity of the strength of the composite to the size of unit cell (or equivalently the size of the inclusions). The work of Ayas et al. (2015) was restricted to shear deformation of the composite with deformation of the elastic inclusion due to mass transport along the matrix/particle interface neglected. Such mass transport is known to become significant (e.g. Zhu et al., 2012) in nickel-based superalloys when the composite is subjected to the more relevant uniaxial tensile stress-state.

The main aim of the present study is to develop a DDP framework which includes interfacial diffusion between two phases such as the matrix/particle interface. Interfacial diffusion in such situations is stress-driven and this presents numerical difficulties in the DDP context due to the singular nature of the stress field within the medium containing a large number of discrete dislocations. In particular, it is critical that the complementary elastic field in the DDP method is accurately estimated at the interface over which diffusion is occurring. Here, we propose a modification to the original superposition framework of Van der Giessen and Needleman (1995) on the lines of that employed by O'Day and Curtin (2004). The outline of the paper is as follows. First we describe the framework used to model interfacial diffusion and the climb-assisted glide DDP framework. Next, we discuss the difficulties of solving the stress-driven interfacial diffusion equations within a DDP framework and detail the superposition framework to overcome these hurdles while still permitting the application of complex boundary conditions such as periodicity. Finally, the DDP predictions are described emphasising the effect of dislocation climb within the matrix, interfacial diffusion and their strong coupling.

\section{Formulation}

DDP has been used extensively to analyse the deformation of composites comprising elastic particles in a single crystal matrix initiated by the pioneering work of Cleveringa et al. (1997). More recent examples include (but not limited to) the studies of Hussein et al. (2008) who analysed the deformation of a voided material and Ayas et al. (2015) who investigated the shear deformation of a composite with elastic inclusions in a single crystal matrix wherein deformation of the matrix was by a combination of dislocation glide and climb. The aim here is to extend the high temperature DDP methodology to incorporate deformation of the composite due to diffusion along the interfaces between the phases of the composite.

We consider a two-dimensional (2D) plane-strain situation with deformation constrained in the $x_{1}-x_{2}$ plane. 
Consistent with the plane-strain condition, only straight edge dislocations perpendicular to the plane of deformation are considered (the $x_{3}$ axis being parallel to the dislocation lines). The 2D composite as sketched in Fig. 1a comprises a single crystal contiguous phase (matrix) and elastic inclusions (or particles). Deformation of the matrix phase is by a combination of glide and climb of dislocations while the particles are assumed to undergo deformation by a combination of bulk elastic distortion and deformation due to interfacial diffusion of the matter making up the particle phase. The inclusion of the interfacial diffusion phenomena requires a modification of the standard DDP methodology of Van der Giessen and Needleman (1995) as employed in previous investigations (Cleveringa et al., 1997; Ayas et al., 2015) and we now proceed to discuss this in two steps. First we describe the interfacial diffusion constitutive framework and then proceed to discuss the DDP methodology required to solve boundary value problems that include interfacial diffusion.

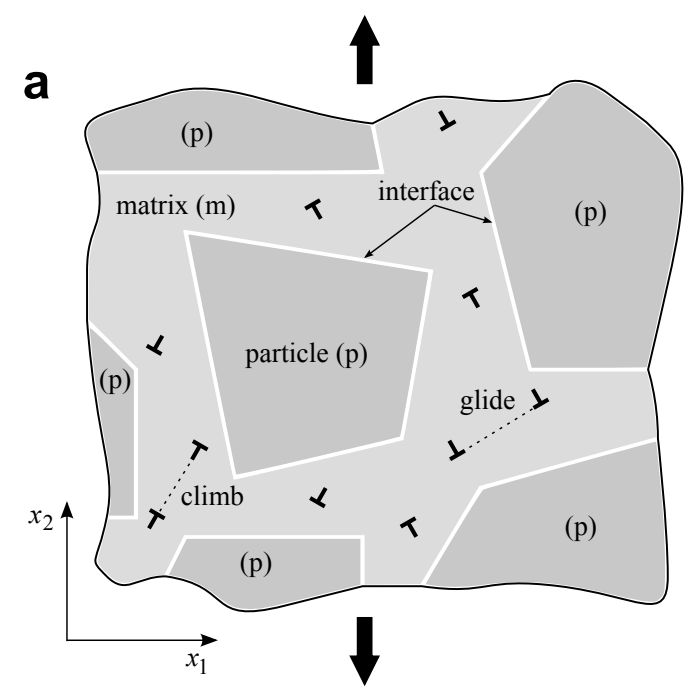

b

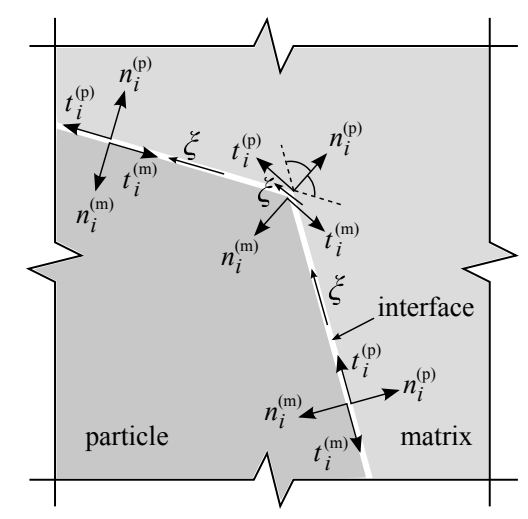

Figure 1: (a) A sketch of multi-phase material comprising particles and a matrix. (b) The sign convention for defining normal and tangential directions at the matrix/particle interfaces; $\xi$ is a local co-ordinate along the interface.

\subsection{Deformation due to diffusion at the interfaces between the particle and matrix}

A gradient in the normal stress on the interface between the particle and matrix provides the driving force for diffusional mass transport of the particle material phase along the interfaces. This has been extensively discussed in the context of the high temperature deformation of metallic and intermetallic matrix composites reinforced by hard particles; e.g. $\gamma$-TiAl reinforced by $\mathrm{Ti}_{2} \mathrm{AlC}$ platelets; see for example Sofronis and McMeeking (1994). This mechanism in high temperature composites has been studied extensively in the literature (e.g. Rosler et al., 1991; Wang and Pan, 2011; Geers et al., 2014) using continuum plasticity to describe the deformation of the matrix phase. We briefly describe here the relevant constitutive framework for this diffusional mass transport. 
The chemical potential $\mu$ per atom of the particle along the matrix/particle interface is given in terms of the atomic volume $\Omega$ as (Needleman and Rice, 1980)

$$
\mu=-\sigma_{\mathrm{n}} \Omega
$$

where $\sigma_{\mathrm{n}} \equiv n_{i}^{(\mathrm{p})} \sigma_{i j}^{(\mathrm{p})} n_{j}^{(\mathrm{p})}$ is the normal stress on the matrix/particle interface with $\sigma_{i j}^{(\mathrm{p})}$ the Cauchy stress and $n_{i}^{(\mathrm{p})}$ the normal to the matrix/particle interface pointing into the matrix as depicted in Fig. 1b. Here, following Needleman and Rice (1980), we have neglected the strain energy contribution to the chemical potential. In the 2D context under consideration here the volumetric flux $j(\xi)$ along the matrix/particle interface is then given as

$$
j(\xi)=\mathscr{D} \frac{\partial \sigma_{\mathrm{n}}(\xi)}{\partial \xi},
$$

where $\xi$ is a local co-ordinate system along the matrix/particle interface as depicted in Fig. $1 \mathrm{~b}$ and the diffusion parameter $\mathscr{D}$ is related to the interfacial diffusion coefficient $D_{\mathrm{b}}$ as well as the effective interface thickness $\delta_{\mathrm{b}}$ via the relation

$$
\mathscr{D}=\frac{D_{\mathrm{b}} \delta_{\mathrm{b}} \Omega}{k T},
$$

where $k$ is the Boltzmann constant and $T$ is the absolute temperature. Mass conservation along the interface then requires that

$$
\frac{\partial}{\partial \xi}\left(\mathscr{D} \frac{\partial \sigma_{\mathrm{n}}(\xi)}{\partial \xi}\right)+\Delta v_{\mathrm{n}}=0,
$$

where $\Delta v_{\mathrm{n}}$ is the normal separation velocity between the particle and the matrix, i.e.

$$
\Delta v_{\mathrm{n}}=-n_{i}^{(\mathrm{p})} \dot{u}_{i}^{(\mathrm{p})}-n_{i}^{(\mathrm{m})} \dot{u}_{i}^{(\mathrm{m})}
$$

where $\dot{u}_{i}^{(\mathrm{p})}$ and $\dot{u}_{i}^{(\mathrm{m})}$ are the material point velocities at adjacent material points on the particle and matrix, respectively. Similar to $n_{i}^{(\mathrm{p})}, n_{i}^{(\mathrm{m})}$ is the normal to the matrix/particle interface pointing into the particle (see Fig. 1b). For simplicity, we also assume that there is perfect bonding between the matrix and particle such that there is no relative sliding at the matrix/particle interface so that

$$
t_{i}^{(\mathrm{p})} \dot{u}_{i}^{(\mathrm{p})}+t_{i}^{(\mathrm{m})} \dot{u}_{i}^{(\mathrm{m})}=0
$$

where $t_{i}^{(\mathrm{p})}$ and $t_{i}^{(\mathrm{m})}$, as depicted in Fig. $1 \mathrm{~b}$, are unit vectors along the matrix/particle interface such that, with $e_{i j k}$ defined to be the permutation tensor, $e_{i j k} n_{j}^{(\mathrm{p})} t_{k}^{(\mathrm{p})}$ and $e_{i j k} n_{j}^{(\mathrm{m})} t_{k}^{(\mathrm{m})}$ are unit vectors in the $x_{3}$ direction. We emphasise that equilibrium dictates traction continuity across the matrix/particle interface such that the tractions $T_{i}^{(\mathrm{p})}=-T_{i}^{(\mathrm{m})}$ with

$$
\begin{aligned}
T_{i}^{(\mathrm{p})} & \equiv \sigma_{i j}^{(\mathrm{p})} n_{j}^{(\mathrm{p})}, \\
T_{i}^{(\mathrm{m})} & \equiv \sigma_{i j}^{(\mathrm{m})} n_{j}^{(\mathrm{m})},
\end{aligned}
$$

and $\sigma_{i j}^{(\mathrm{p})}$ and $\sigma_{i j}^{(\mathrm{m})}$ are the Cauchy stresses at adjacent material points in the particle and matrix, respectively. 


\subsection{Discrete dislocation methodology}

The basic two-dimensional DDP framework of Van der Giessen and Needleman (1995) is used to solve the boundary value problem. However, the inclusion of the interfacial diffusion requires a key modification to this framework which is described in Section 2.2.1.

In DDP, the dislocations are treated as line defects in an otherwise elastic continuum with their collective motion occurring by a combination of glide and climb resulting in plastic deformation. The stress state and deformation are computed using superposition of a singular field ( ()) that captures the elastic singularities associated with the dislocations and a smooth image field ( $\left(^{\wedge}\right)$ that enforces the boundary conditions (Van der Giessen and Needleman, 1995). The computation of the deformation history of a body is performed in an incremental manner due to the combined climb/glide motion of dislocations as follows. The dislocation structure, the stress state and thus the velocity discontinuity at matrix/particle interfaces are assumed to be known at a given instant. A loading increment is prescribed and (i) the deformation and stress states are updated, and (ii) the evolution of the dislocation structure is computed. This involves four main computations at each time increment:

(a) determining the glide and climb Peach-Koehler ( $\mathrm{P}-\mathrm{K})$ forces on the dislocations from the known deformation state;

(b) determining the rate of change of the dislocation configuration caused by the motion of dislocations, generation of new dislocations, their mutual annihilation and their possible pinning and release from obstacles;

(c) determining the displacement, stress and strain states for the updated dislocation arrangement by solving a complementary boundary value problem; and

(d) determining the rate of displacement jump at the matrix/particle interfaces via Eq. (4).

The complementary boundary value problem follows from the superposition recipe proposed by Van der Giessen and Needleman (1995) and briefly summarised here. At a given stage of loading, the body contains $N$ edge dislocations and the material velocity, strain rate and stress rate fields are written as the superposition of two fields

$$
\dot{u}_{i}=\dot{\hat{u}}_{i}+\dot{\tilde{u}}_{i}, \quad \dot{\varepsilon}_{i j}=\dot{\hat{\varepsilon}}_{i j}+\dot{\tilde{\varepsilon}}_{i j}, \quad \dot{\sigma}_{i j}=\dot{\hat{\sigma}}_{i j}+\dot{\tilde{\sigma}}_{i j},
$$

where the ( $)$ fields are the sum of all the contributions of the individual dislocations, viz.

$$
\dot{\tilde{u}}_{i}=\sum_{I=1}^{N} \dot{\tilde{u}}_{i}^{(I)}, \quad \dot{\tilde{\varepsilon}}_{i j}=\sum_{I=1}^{N} \dot{\tilde{\varepsilon}}_{i j}^{(I)}, \quad \dot{\tilde{\sigma}}_{i j}=\sum_{I=1}^{N} \dot{\tilde{\sigma}}_{i j}^{(I)} .
$$

These ( $)$ ) fields give rise to traction rates $\dot{\tilde{T}}_{i}$ (三 $\dot{\tilde{\sigma}}_{i j} n_{j}$ ) and material velocities $\dot{\tilde{u}}_{i}$ on the boundary of the body. The (^) fields correct for the required boundary conditions and are obtained by solving a complementary boundary value problem using the finite element (FE) method. Note that typically we calculate $\dot{\tilde{u}}_{i}^{(I)}$ for dislocation (I) from time $t$ to time $t+\Delta t$ using a finite difference scheme by decomposing the combined motion of dislocations into two sequential glide-only and climb-only components as described in Danas and Deshpande (2013). 


\subsubsection{Imposition of the velocity discontinuity across matrix/particle interfaces}

Interfacial diffusion results in a velocity discontinuity as prescribed by Eq. (5) across the matrix/particle interface along with traction continuity. Imposing these boundary conditions within the DDP superposition framework requires some special considerations due to the singular nature of the fields within the medium. We first elaborate on the numerical difficulties and then present their resolution.

For simplicity, consider the problem comprising a plastic matrix with elastic particles (i.e. no dislocations within the particles). The DDP framework for this problem was first introduced by Cleveringa et al. (1997) where the field quantities in the "entire" domain (i.e. both matrix and particle phases) are written as a superposition of the ( () and (^) fields as described above. For the case of no interfacial diffusion with perfect bonding between the particle and matrix, there is both traction as well as displacement continuity across the matrix/particle interface and the superposition framework can be used to accurately determine the fields near the interface in both the matrix and particle. Now consider the problem where interfacial diffusion is permitted such that there is a displacement discontinuity at the interface along with traction continuity as defined in Section 2.1. Dislocations in the matrix give rise to singular (and discontinuous) displacement fields in the matrix phase. However, the discontinuous nature of the displacement fields across the matrix/particle interface implies that these singularities are significantly relaxed by the interfacial diffusion (and consequent displacement discontinuity) along the matrix/particle interface. If a superposition principle as employed by Cleveringa et al. (1997) or Ayas et al. (2015) is applied, the ( $)$ fields across the matrix/particle interface are continuous and the large change in the total stress across the interface will need to be accounted for in the (^) fields. However, recall that the (^) fields are typically calculated by a FE procedure that is unsuited to resolve such large gradients and this then leads to inaccurate estimates of the (^) fields near the matrix/particle interface.

A solution to this problem is to modify the superposition as follows: (i) the total fields of the matrix phase are written as a superposition of the ( $\left(^{()}\right.$fields of the dislocations within the matrix phase and the (^) fields that correct for the boundary conditions (and typically are calculated numerically via an FE analysis) and (ii) the total fields within the particle phase (which does not contain any dislocations) are given only by the so-called ( $\left.{ }^{(}\right)$fields that are calculated via an FE calculation. This means that the numerically calculated fields within the particle now do not need to correct for singularities present in the matrix phase. This idea carries forward to when dislocations on different slip systems are present within both the particle and the matrix. Then, singularities (and displacement discontinuities) associated with dislocations are not transmitted from the matrix into the particle and vice versa. Thus, in general, the total fields within the matrix and particle phases are written as

$$
\dot{u}_{i}^{(\mathrm{m})}=\dot{\hat{u}}_{i}^{(\mathrm{m})}+\dot{\tilde{u}}_{i}^{(\mathrm{m})}, \quad \dot{\varepsilon}_{i j}^{(\mathrm{m})}=\dot{\hat{\varepsilon}}_{i j}^{(\mathrm{m})}+\dot{\tilde{\varepsilon}}_{i j}^{(\mathrm{m})}, \quad \dot{\sigma}_{i j}^{(\mathrm{m})}=\dot{\hat{\sigma}}_{i j}^{(\mathrm{m})}+\dot{\tilde{\sigma}}_{i j}^{(\mathrm{m})},
$$

and

$$
\dot{u}_{i}^{(\mathrm{p})}=\dot{\hat{u}}_{i}^{(\mathrm{p})}+\dot{\tilde{u}}_{i}^{(\mathrm{p})}, \quad \dot{\varepsilon}_{i j}^{(\mathrm{p})}=\dot{\hat{\varepsilon}}_{i j}^{(\mathrm{p})}+\dot{\tilde{\varepsilon}}_{i j}^{(\mathrm{p})}, \quad \dot{\sigma}_{i j}^{(\mathrm{p})}=\dot{\hat{\sigma}}_{i j}^{(\mathrm{p})}+\dot{\tilde{\sigma}}_{i j}^{(\mathrm{p})},
$$

respectively. Here, the ()$^{(\mathrm{m})}$ and $\left(^{()^{(\mathrm{p})}}\right.$ are the sum of all the contributions of the individual dislocations within the matrix and particle, respectively. This modified superposition framework is schematically shown in Fig. 2 with ap- 


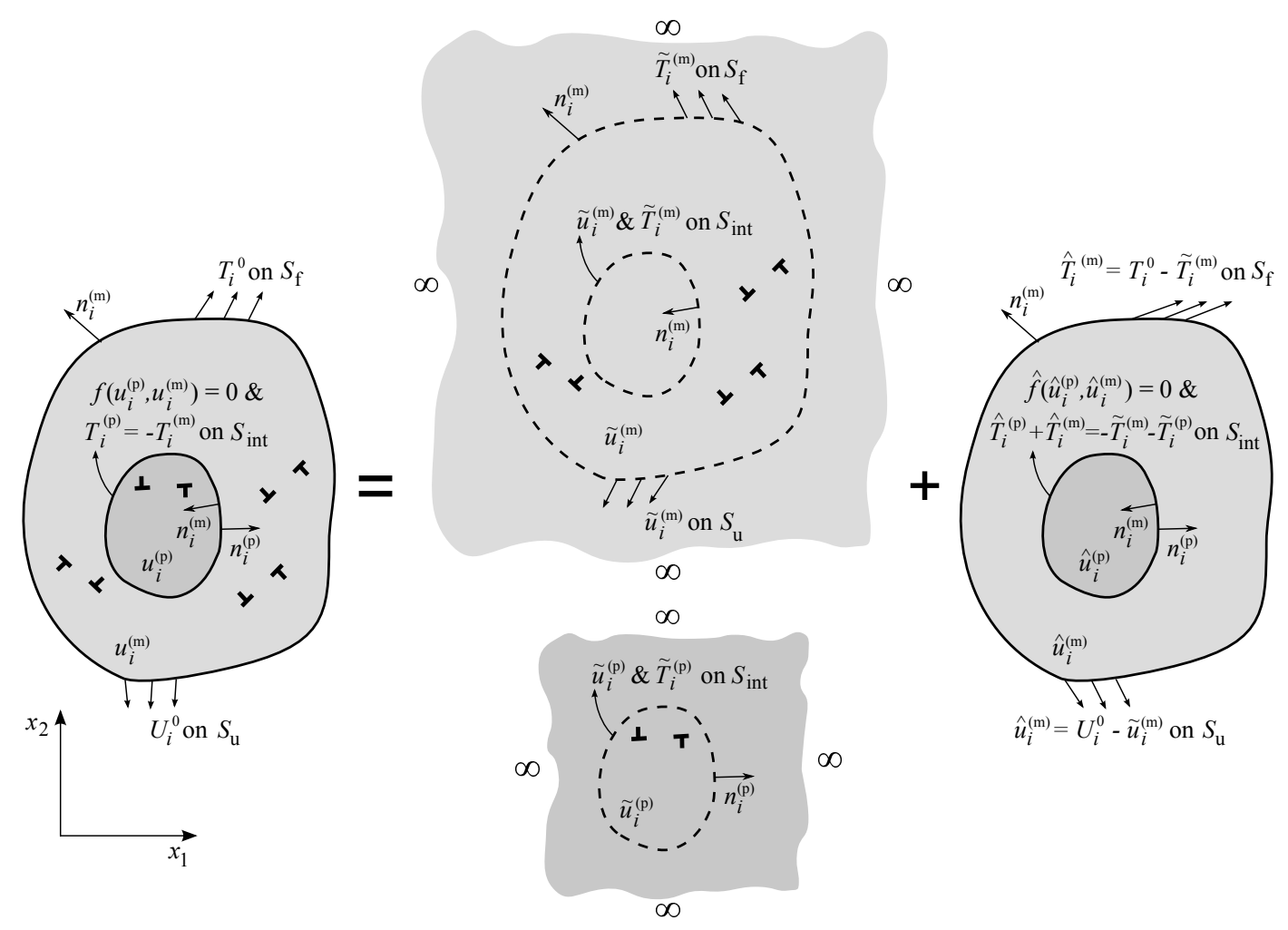

Figure 2: Sketch illustrating the superposition principle used to model the deformation of a multi-phase medium with particulate inclusions, including the effect of interfacial diffusion between the matrix and particles. For the sake of brevity the velocity discontinuity conditions at the matrix/particle interfaces due to interfacial diffusion are represented in generic forms $f\left(u_{i}^{(\mathrm{p})}, u_{i}^{(\mathrm{m})}\right)=0$ and $\hat{f}\left(\hat{u}_{i}^{(\mathrm{p})}, \hat{u}_{i}^{(\mathrm{m})}\right)=0$ for the total and $\left(^{\wedge}\right)$ fields, respectively.

propriate illustrations of the boundary conditions at the external boundaries as well as at the matrix/particle interfaces. Each of the (־) fields gives rise to the tractions and displacements at the associated boundaries as illustrated in Fig. 2. Hence, the imposition of the displacement discontinuity due to interfacial diffusion at the matrix/particle interface reduces to the constraint equations in terms of the (^) fields via Eqs. (5) and (6) to

$$
\begin{aligned}
n_{i}^{(\mathrm{p})} \dot{\hat{u}}_{i}^{(\mathrm{p})}+n_{i}^{(\mathrm{m})} \dot{\hat{u}}_{i}^{(\mathrm{m})} & =-\Delta v_{\mathrm{n}}-n_{i}^{(\mathrm{m})} \dot{\tilde{u}}_{i}^{(\mathrm{m})}-n_{i}^{(\mathrm{p})} \dot{\tilde{u}}_{i}^{(\mathrm{p})}, \\
t_{i}^{(\mathrm{p})} \dot{\hat{u}}_{i}^{(\mathrm{p})}+t_{i}^{(\mathrm{m})} \dot{\hat{u}}_{i}^{(\mathrm{m})} & =-t_{i}^{(\mathrm{m})} \dot{\tilde{u}}_{i}^{\mathrm{m})}-t_{i}^{(\mathrm{p})} \dot{\tilde{u}}_{i}^{(\mathrm{p})},
\end{aligned}
$$

respectively, and traction continuity implies $\hat{T}_{i}^{(\mathrm{p})}+\hat{T}_{i}^{(\mathrm{m})}=-\tilde{T}_{i}^{(\mathrm{m})}-\tilde{T}_{i}^{(\mathrm{p})}$ where, analogous to Eq. (7), the (^) tractions are defined as

$$
\begin{aligned}
\hat{T}_{i}^{(\mathrm{p})} & \equiv \hat{\sigma}_{i j}^{(\mathrm{p})} n_{j}^{(\mathrm{p})}, \\
\tilde{T}_{i}^{(\mathrm{p})} & \equiv \tilde{\sigma}_{i j}^{(\mathrm{p})} n_{j}^{(\mathrm{p})}, \\
\hat{T}_{i}^{(\mathrm{m})} & \equiv \hat{\sigma}_{i j}^{(\mathrm{m})} n_{j}^{(\mathrm{m})}, \\
\tilde{T}_{i}^{(\mathrm{m})} & \equiv \tilde{\sigma}_{i j}^{(\mathrm{m})} n_{j}^{(\mathrm{m})} .
\end{aligned}
$$

We note in passing here that this modification to the standard superposition method implies that no polarisation 
correction is required in the solution of the (^) fields when there is an elastic property mismatch between the matrix and particles phases. Inclusion of the polarisation term is required in such cases in the original superposition principle of Van der Giessen and Needleman (1995) as observed by the authors.

\subsubsection{Dislocation interaction constitutive rules}

While the long-range interactions of dislocations are captured by the linear elastic fields, the short-range interactions are incorporated through a set of constitutive rules which were given by Kubin et al. (1992) for glide-only motion of dislocations and subsequently employed by Van der Giessen and Needleman (1995) in plane-strain analyses. Here, we allow for both glide and climb motion of dislocations and discuss the constitutive rules with an emphasis on the differences with respect to the glide-only case. With dislocations allowed to both glide and climb, they are no longer confined to a fixed slip plane and the basic entity is a slip system (i.e. the orientation in the lattice of the slip plane normal and the slip direction). The glide and climb force work conjugates to infinitesimal variations of the position of dislocation $I$ (i.e. the glide and climb P-K force) are given by

$$
\begin{aligned}
& f_{\mathrm{g}}^{(I)}=m_{i}\left(\hat{\sigma}_{i j}+\sum_{J \neq I} \tilde{\sigma}_{i j}^{(J)}\right) b_{j}^{(I)}, \quad(J=1, \cdots, N), \\
& f_{\mathrm{c}}^{(I)}=-s_{i}\left(\hat{\sigma}_{i j}+\sum_{J \neq I} \tilde{\sigma}_{i j}^{(J)}\right) b_{j}^{(I)}, \quad(J=1, \cdots, N),
\end{aligned}
$$

respectively, where $b_{j}^{(I)}$ is the Burgers vector of dislocation $I$ residing on a slip system with unit normal $m_{i}$ and a unit vector $s_{i}$ in the slip direction such that $e_{i j k} s_{j} m_{k}$ is a unit vector in the $x_{3}$ direction.

The glide velocity of dislocation $I$ is defined by the usual drag relation $v_{\mathrm{g}}^{(I)}=f_{\mathrm{g}}^{(I)} / B_{\mathrm{g}}$ with $B_{\mathrm{g}}$ the drag coefficient in glide. In most DDP computations, an explicit time integration scheme is employed for the dislocation dynamics computations with a time step $\Delta t$. Such an explicit scheme tends to introduce artificial oscillations into the glide motion of dislocations when the spatial gradients in $f_{\mathrm{g}}^{(I)}$ are large. Thus, following Chakravarthy and Curtin (2011), a semi-implicit scheme is adopted to calculate the glide velocity of dislocations such that

$$
v_{\mathrm{g}}^{(I)}=\frac{1}{B_{\mathrm{g}}} \frac{f_{\mathrm{g}}^{(I)}}{\left(1-\frac{1}{B_{\mathrm{g}}} \frac{\partial f_{\mathrm{g}}^{(I)}}{\partial \zeta_{\mathrm{g}}} \Delta t\right)},
$$

where $\zeta_{\mathrm{g}}$ is a local co-ordinate along the glide direction of dislocation. Following Danas and Deshpande (2013), the drag relation for the climb velocity is similarly taken to have the form $v_{\mathrm{c}}^{(I)}=f_{\mathrm{c}}^{(I)} / B_{\mathrm{c}}$ with $B_{\mathrm{c}}$ the so-called climb drag coefficient. The climb rate of dislocations is several orders of magnitude lower than the glide rate (i.e., $B_{\mathrm{c}} \gg B_{\mathrm{g}}$ ), and hence oscillations in the climb motion of the dislocations are negligible. It thus suffices to update the climb motion of the dislocation in an explicit manner over the time step $\Delta t$ using the climb drag relation detailed above.

The glide component of the P-K force also controls the nucleation of dislocations by a $2 \mathrm{D}$ representation of the Frank-Read source. This source is treated as a point that generates a dipole of edge dislocations when the glide P-K force at the source exceeds the source strength $\tau_{\text {nuc }} b$ during a time period $t_{\text {nuc }}$ (Van der Giessen and Needleman, 1995), 
where $b$ is the magnitude of the Burgers vector. The Burgers vector of dislocations is aligned with $s_{i}$ and their sign is determined according to the direction of the glide P-K force at the position of the source. The distance $L_{\text {nuc }}$ between the dislocations of the nucleated dipole is chosen in such a way that the total resolved shear stress on the slip plane balances the attractive shear stress that the two opposite signed dislocations exert on each other. Note that we neglect climb of the nascent dislocation loop.

Annihilation of two opposite signed dislocations on the same slip system occurs when they are sufficiently close together. Within the present framework, this is modeled by eliminating the dislocations when they are within a material-dependent critical annihilation distance $L_{\mathrm{e}}$. Obstacles to dislocation motion are modeled as points associated with a slip system. An obstacle pins dislocations on the same slip system that pass within a specified distance, also taken to be $L_{\mathrm{e}}$, as long as the P-K force at the obstacle position is below the obstacle strength $\tau_{\mathrm{obs}} b$.

\section{The composite boundary value problem}

We consider an infinitely large periodic composite material subjected to uniaxial tensile loading as shown in the sketch in Fig. 3. The composite comprises an elastic/plastic single crystal matrix with a Young's modulus $E$ and Poisson ratio $v$ within which elastic particles are embedded. The elastic mismatch between the matrix and particles is neglected so that the particles also have the same Young's modulus $E$ and Poisson ratio $v$. Dislocation nucleation and motion takes place within the matrix on the slip systems oriented at angles $\varphi^{(1)}=\varphi$ and $\varphi^{(2)}=180^{\circ}-\varphi$ with respect to the $x_{1}$ axis, respectively (cf. Fig. 3), where $\varphi=30^{\circ}$. The elastic particles are assumed to be completely impenetrable to dislocations and deform by a combination of elastic deformation and interfacial diffusion as described in Section 2.1.

The periodic composites have a volume fraction $V_{f}$ of elastic particles and here we analyse a rectangular unit cell of dimensions $(h / \tan \varphi) \times h$ as shown in Fig. 3. The aspect ratio of the unit cell ensures periodicity of the motion of the dislocations on two slip systems, i.e. dislocations exiting from one side of the unit cell re-enter from the corresponding point on the opposite side. The rectangular elastic particles of size $w^{(\mathrm{p})} \times h^{(\mathrm{p})}$ are arranged in a regular cubic arrangement in the unit cell as shown in Fig. 3 such that $V_{f} \approx 46.6 \%$ with $h^{(\mathrm{p})} / w^{(\mathrm{p})}=9 / 7$. This aspect ratio of the elastic particles was chosen to ensure that (i) no dislocations in the absence of dislocation climb can continuously traverse the matrix without getting blocked by an elastic particle, and (ii) the matrix surrounding the particles has a more or less uniform thickness, i.e. the vertical and horizontal matrix channels are of approximately equal widths.

Loading is imposed by specifying periodic boundary conditions on the edges of the unit cell such that in terms of the imposed macroscopic strain rate $\dot{\bar{\varepsilon}}_{i j}$, we have

$$
\Delta \dot{u}_{i}=\dot{\bar{\varepsilon}}_{i j} \Delta x_{j}
$$

where $\Delta \dot{u}_{i}$ is the difference between displacement rates on opposite sides of the unit cell specified by the difference in position vector $\Delta x_{j}$. Moreover, periodicity dictates that the tractions $T_{i}=\sigma_{i j} n_{j}$ (with $n_{j}$ the outward unit normal to 


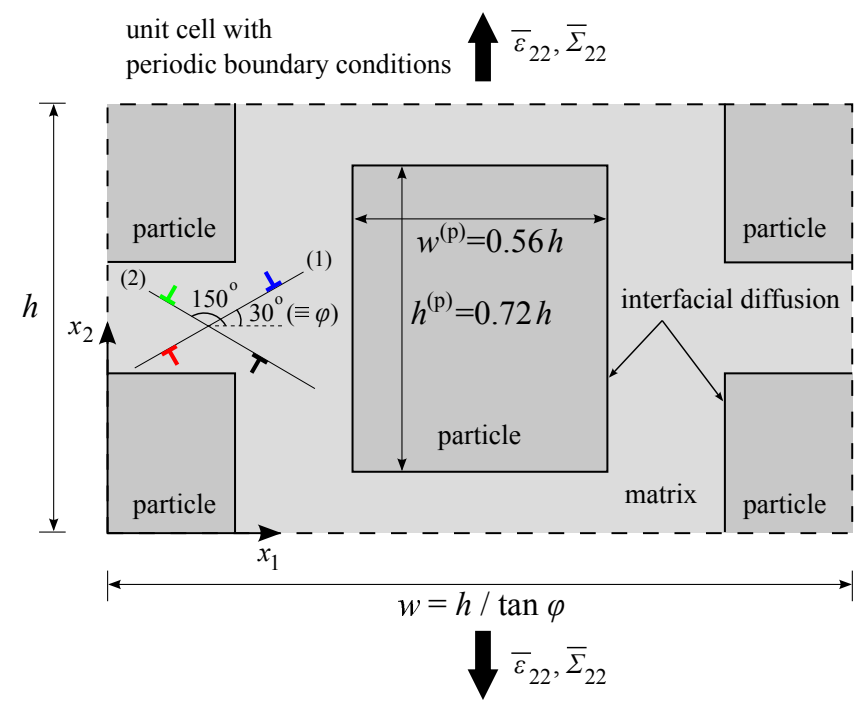

Figure 3: Sketch of the unit cell of the composite analysed in this study. The rectangular unit comprises rectangular elastic particles in an elastic/plastic matrix and is subjected to uniaxial tensile loading in the $x_{2}$ direction. The two active slip systems within the matrix are illustrated along with the positive and negative edge dislocations on these slip systems: the colours used to depict these dislocations are utilised to illustrate dislocation structures in the remainder of the paper.

the unit cell) at these two locations separated by $\Delta x_{j}$ are equal and opposite. The corresponding macroscopic stresses $\bar{\Sigma}_{i j}$ which are work conjugates to $\bar{\varepsilon}_{i j}$ are given as

$$
\bar{\Sigma}_{i j}=\frac{\tan \varphi}{2 h^{2}} \int_{C}\left(T_{i} x_{j}+T_{j} x_{i}\right) \mathrm{d} C
$$

where $C$ is the boundary of the unit cell. Here, we only consider uniaxial loading in the $x_{2}$ direction by imposing a strain rate $\dot{\bar{\varepsilon}}_{22}=\dot{\varepsilon}_{\text {app }}$ with $\bar{\varepsilon}_{11}$ and $\bar{\varepsilon}_{12}$ being solution variables that ensure that $\bar{\Sigma}_{11}=\bar{\Sigma}_{12}=0$. The specification of the boundary conditions is then completed by imposing the velocity discontinuities at the matrix/particle interfaces as given by Eqs. (5) and (6).

\subsection{Numerical solution methodology}

The basic numerical solution technique for DDP problems is detailed in numerous previous studies; see for example (Cleveringa et al., 1999; Segurado et al., 2007; Chakravarthy and Curtin, 2011; Shishvan et al., 2011). Here, we focus on providing some details on numerical techniques specific to the interfacial diffusion that is included in the problem analysed here.

Recall that there are no dislocations in the particle in the problem analysed here and thus the ( ${ }^{(\mathrm{p})}$ fields vanish. Therefore, the constraint equations (12) for the (^) fields are of the form

$$
\begin{aligned}
n_{i}^{(\mathrm{p})} \dot{\hat{u}}_{i}^{(\mathrm{p})}+n_{i}^{(\mathrm{m})} \dot{\hat{u}}_{i}^{(\mathrm{m})} & =-\Delta v_{\mathrm{n}}-n_{i}^{(\mathrm{m})} \dot{\tilde{u}}_{i}^{(\mathrm{m})}, \\
t_{i}^{(\mathrm{p})} \hat{\hat{u}}_{i}^{(\mathrm{p})}+t_{i}^{(\mathrm{m})} \dot{\hat{u}}_{i}^{(\mathrm{m})} & =-t_{i}^{(\mathrm{m})} \dot{\tilde{u}}_{i}^{(\mathrm{m})},
\end{aligned}
$$


while traction continuity along the matrix/particle interface reduces to $\hat{T}_{i}^{(\mathrm{p})}+\hat{T}_{i}^{(\mathrm{m})}=-\tilde{T}_{i}^{(\mathrm{m})}$. In the context of FE analysis, the so-called master-slave method is adopted to satisfy these constraints and imposed at the nodes of the FE meshes as displacement and force constraints, respectively. Calculation of the nodal forces at the interface due to the matrix dislocations requires special consideration due to the large stress gradients associated with dislocations in the matrix near the interface. To accurately evaluate these forces, (i) we use trapezoidal integration of $\tilde{\sigma}_{i j}^{(\mathrm{m})}$ along the element boundaries with 32 integration points and (ii) dislocations that are blocked by the particles sit with a stand-off distance, $L_{\mathrm{e}}$, from the interfaces. It is noted that a core cut-off radius, $L_{\mathrm{e}}$, is also used around dislocations while calculating their displacement/stress fields.

The calculation of the interfacial velocity discontinuity requires first the computation of $\sigma_{\mathrm{n}}$ and therefore the normals to the interface must be established. These normals are not defined at the corners of the particles and there we take $n_{i}^{(\mathrm{p})}$ to lie along the vector that bisects the internal angle of the corner of the particle with $t_{i}^{(\mathrm{p})}$ again defined so that $e_{i j k} n_{j}^{(\mathrm{p})} t_{k}^{(\mathrm{p})}$ is a unit vector in the $x_{3}$ direction. The definitions of $n_{i}^{(\mathrm{m})}$ and $t_{i}^{(\mathrm{m})}$ follow analogously as depicted in Fig. $1 \mathrm{~b}$. With $\sigma_{\mathrm{n}}$ known, $\Delta v_{\mathrm{n}}$ requires the computation of the second spatial derivative of $\sigma_{\mathrm{n}}$ with respect to the spatial coordinate $\xi$ along the matrix/particle interface. This spatial derivative is computed using the central difference operator as follows. The interface is divided into segments of equal length $\Delta \xi=\left\|\xi_{j+1}-\xi_{j}\right\|_{2}$ where $\xi_{j}$ is the co-ordinate of the $j^{\text {th }}$ FE node along the local $\xi$ axis and \|\|$_{2}$ denotes the distance norm. Then, using the central difference operator, the normal velocity discontinuity at position $\xi_{j}$ is written via Eq. (4) as

$$
\Delta v_{\mathrm{n}}\left(\xi_{j}\right)=-\mathscr{D} \frac{\left(-\sigma_{\mathrm{n}}\left(\xi_{j+2}\right)+16 \sigma_{\mathrm{n}}\left(\xi_{j+1}\right)-30 \sigma_{\mathrm{n}}\left(\xi_{j}\right)+16 \sigma_{\mathrm{n}}\left(\xi_{j-1}\right)-\sigma_{\mathrm{n}}\left(\xi_{j-2}\right)\right)}{12(\Delta \xi)^{2}}
$$

and the velocities of the corresponding matrix and particle material points are given by Eq. (5).

The imposition of the periodic boundary conditions is carried out in the FE calculations following the basic idea introduced in Hussein et al. (2008). Briefly, the individual dislocation fields are calculated analytically from the known closed-form solution of dislocations in an infinite medium. Thus, the ( $\left(^{\sim}\right)$ fields are not periodic and periodicity is enforced via the (^) fields, such that the total fields from the superposition of ( $\left(^{()}\right.$and (^) fields are periodic. We also impose periodicity on the motion of the dislocations, such that dislocations leaving from one side of the unit cell re-enter from the opposite side. This is achieved by removing the exiting dislocations from the calculation and re-inserting a new dislocation at the corresponding point on the opposite side of the unit cell. Recall that the rate forms of the ( ) displacement fields are used here and thus the slip contribution of the dislocations is automatically retained when dislocations are removed from one side of the unit cell and reintroduced from the opposite side.

Resolving the dislocation dynamics requires a small time step of $\Delta t=0.5 \mathrm{~ns}$; correspondingly, all calculations have been carried out with a rather high applied strain rate of $\dot{\varepsilon}_{\text {app }}=2000 \mathrm{~s}^{-1}$ in order to limit the computational time. This artificially high strain rate implies that we need to scale not only the climb rate of dislocations but also the interfacial diffusion parameter $\mathscr{D}$ so as to keep the ratio of the diffusion time to loading time in approximately the appropriate regime seen in experiments. While the scaling of the vacancy diffusion rate is addressed in (Ayas et al., 2014) the scaling of the interfacial diffusion constant is explained in the subsequent section. A finite element mesh of 
approximately square bi-linear elements is used where the mesh size is $\sim h / 50$ (except for the smallest size of the unit cell in which case the element size is $\sim h / 25)$.

\subsection{Reference parameters}

The matrix and particles are elastically isotropic with $E=100 \mathrm{GPa}$ and $v=0.37$. Plasticity is constrained to the matrix which is initially dislocation-free but contains randomly distributed dislocation sources with density $\rho_{\text {nuc }} \simeq 300 \mu \mathrm{m}^{-2}$, and obstacles, with density $\rho_{\mathrm{obs}} \simeq 600 \mu \mathrm{m}^{-2}$. These densities were chosen following Hussein et al. (2008) and Ayas et al. (2015) so as to ensure that the matrix in the absence of particles has negligible strain hardening for the unit cell sizes considered in this study. The strength of each source is selected from a Gaussian distribution with an average of $\bar{\tau}_{\text {nuc }}=50 \mathrm{MPa}$ and standard deviation of $1 \mathrm{MPa}$ while all obstacles have a strength $\tau_{\mathrm{obs}}=150 \mathrm{MPa}$. The nucleation time for all sources is taken as $t_{\text {nuc }}=10 \mathrm{~ns}$. To illustrate the inevitable statistical effects in DDP calculations, we consider at least five realisations of source/obstacle locations and random source strengths for each size of the unit cell.

Dislocations generated from the sources are assigned a Burgers vector with a magnitude $b=0.25 \mathrm{~nm}$. While the glide drag coefficient for dislocation motion is $B_{\mathrm{g}}=10^{-4} \mathrm{~Pa} \mathrm{~s}$, the climb drag coefficient $B_{\mathrm{c}}$ is taken as a parameter to investigate the effect of dislocation climb rates on the plastic response of the composites. Unless otherwise specified, results are presented for the "glide-only" limit of $B_{\mathrm{c}} / B_{\mathrm{g}}=\infty$ and a reference "climb-assisted glide" value of $B_{\mathrm{c}} / B_{\mathrm{g}}$. This reference value is chosen using the temporal scaling argument presented in Ayas et al. (2014) which requires that the ratio $\bar{\tau}$ of the vacancy diffusion time and loading time in the simulations and experiments is equal ${ }^{1}$. Now consider a representative experiment with the vacancy diffusion co-efficient $D_{\mathrm{v}}=10^{-5} \mu \mathrm{m}^{2} s^{-1}$ and an applied strain rate of $10^{-7} \mathrm{~s}^{-1}$ which gives $\bar{\tau}=100 \rho_{\mathrm{dis}}$ in the experiments in terms of the dislocation density $\rho_{\mathrm{dis}}\left(\right.$ in $\mu \mathrm{m}^{-2}$ ). With the dislocation density equal in the experiments and simulations, this implies a scaled vacancy diffusion co-efficient of $D_{\mathrm{v}}=2 \times 10^{5} \mu \mathrm{m}^{2} \mathrm{~s}^{-1}$ for $\dot{\varepsilon}_{\mathrm{app}}=2000 \mathrm{~s}^{-1}$ in the simulations. Using the relation between $B_{\mathrm{c}}$ and $D_{\mathrm{v}}$ from Danas and Deshpande (2013) we set a reference value of $B_{\mathrm{c}} / B_{\mathrm{g}}=10^{3}$.

It now remains to specify the interfacial diffusion constant $\mathscr{D}$. We present parametric studies to investigate its effect in terms of the non-dimensional group

$$
\mathscr{D}^{*}=\frac{\mathscr{D} \bar{\tau}_{\text {nuc }}}{\left(1 / \sqrt{\rho_{\text {nuc }}}\right)^{3} \dot{\varepsilon}_{\text {app }}} .
$$

This group represents the ratio of the time for diffusion to occur along the interface and the loading time; an equivalent form of this group in the continuum plasticity limit was used by Sofronis and McMeeking (1994) in their investigation of creep of metal matrix composites. It is instructive to develop an understanding of physically realistic values of this non-dimensional parameter $\mathscr{D}^{*}$. For this purpose we consider a nickel-based superalloy comprising $\gamma^{\prime}$ second phase

\footnotetext{
${ }^{1}$ The diffusion time of the vacancies is $\approx R^{2} / D_{\mathrm{v}}$ with the dislocation spacing $R$ related to the dislocation density $\rho_{\text {dis }}$ via $R \approx 1 / \sqrt{\rho_{\text {dis }}}$, while the loading time is $1 / \dot{\varepsilon}_{\text {app }}$.
} 
particles in a $\gamma$ phase matrix. Tensile experiments are typically conducted on such materials with strain rates on the order $\dot{\varepsilon}_{\text {app }}=10^{-6} \sim 10^{-8} \mathrm{~s}^{-1}$ and interfacial diffusion in these materials is due to the diffusion of $\mathrm{Al}$ within the $\mathrm{Ni}$ lattice. The tracer diffusion co-efficient $D_{\mathrm{b}}$ of $\mathrm{Al}$ in $\mathrm{Ni}$ is in the range $10^{-4}$ to $10^{-6} \mu \mathrm{m}^{2} \mathrm{~s}^{-1}$ for temperatures in the range $T=1200$ to $1400 \mathrm{~K}$ (Ikeda et al., 1998). Then substituting these values in Eqs. (3) and (21) with the atomic volume $\Omega$ of $\mathrm{Al}$ and interface thickness $\delta_{\mathrm{b}}$ taken to be $13 \times 10^{-12} \mu \mathrm{m}^{3}$ and $1 \mathrm{~nm}$, respectively, we have $\mathscr{D}^{*}$ to lie in the range $\sim 0.2-20$. The applied strain rates in the simulations are significantly higher than $10^{-6} \mathrm{~s}^{-1}$ but we keep $\mathscr{D}^{*}$ to lie in this range so as to accurately capture the effect of interfacial diffusion over the experimental time-scales. Unless otherwise stated, reference results are presented for the case of $\mathscr{D}^{*}=0.65$ and compared with the results obtained for $\mathscr{D}^{*}=0$, i.e. when the interfacial diffusion is absent. In addition, parametric studies for the effect of interfacial diffusion constant over the range $0.13<\mathscr{D}^{*}<6.5$ will also be reported.

\section{DDP predictions of the tensile response of the composite}

We now proceed to present numerical predictions for the tensile response of the composite based on the formulation and material properties detailed above. The results will be presented in two parts. First we restrict plasticity in the matrix to occur by only the glide motion of dislocations and then contrast this when plasticity in the matrix is via climb-assisted glide motion of dislocations.

\subsection{Glide-only motion of dislocations}

DDP predictions of the tensile response of composites with unit cell sizes varying from $h=0.25 \mu \mathrm{m}$ to $2 \mu \mathrm{m}$ are plotted in Fig. 4 for glide-only motion of dislocations and the two reference values of $\mathscr{D}^{*}$. In Fig. 4a results are presented for the normalised applied stress $\bar{\sigma}_{22} \equiv \bar{\Sigma}_{22} / \bar{\tau}_{\text {nuc }}$ versus the work-conjugate strain $\bar{\varepsilon}_{22}$ while in Fig. $4 \mathrm{~b}$ the corresponding evolution of the dislocation density $\rho_{\mathrm{dis}}$ with $\bar{\varepsilon}_{22}$ is plotted. Here $\rho_{\mathrm{dis}}$ is defined as the ratio of the number of dislocations to the area of the matrix material in the unit cell (recall dislocations are only present within the matrix). In all cases the results shown are averages over at least five realisations of the initial source and obstacle distributions within the unit cell.

First consider the $\mathscr{D}^{*}=0$ case. After an initial elastic response all the sizes of the unit cells of the composites analysed here display a hardening plastic response with the hardening rate increasing with decreasing $h$. By contrast, the evolution of the dislocation density is nearly independent of the unit cell size. To understand these responses of the composite it is instructive to visualise the deformation fields within it. Recall that the displacement field $u_{i}$ in DDP is discontinuous as dislocation glide gives rise to a displacement discontinuity across the slip plane. Thus, a measure of strain which includes this slip contribution cannot be defined. Rather, here we define a strain-like measure with the sole purpose of visualising the deformation. This is done as follows. We first evaluate $u_{i}$ on a uniform square grid of spacing $0.01 \mu \mathrm{m}$ (this grid is different from the FE mesh). Then, a measure of strain $E_{i j}=0.5\left(u_{i, j}+u_{j, i}\right)$ is numerically evaluated by performing the required spatial differentiations using bi-linear interpolation functions for $u_{i}$ and slip on 

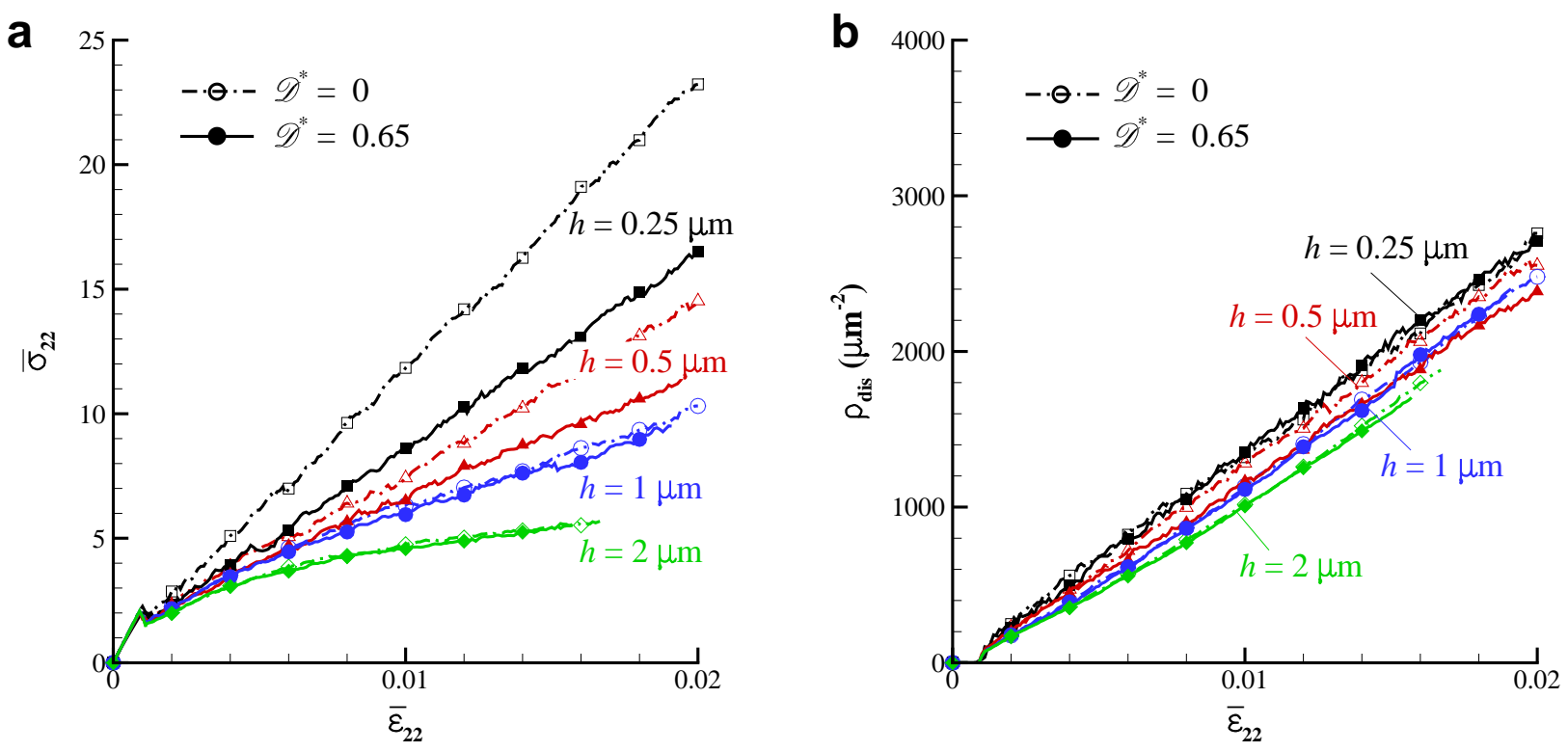

Figure 4: (a) The normalised tensile stress $\bar{\sigma}_{22}$ versus strain $\bar{\varepsilon}_{22}$ responses of composites with glide-only motion of dislocations $\left(B_{\mathrm{c}} / B_{\mathrm{g}}=\infty\right)$ and (b) the corresponding evolution of dislocation density $\rho_{\text {dis }}$ in the matrix. Results are shown for four unit cell sizes $h$ as well as for interfacial diffusion constants $\mathscr{D}^{*}=0$ and 0.65 .

system $(\beta)$ then calculated as $\gamma^{(\beta)}=s_{i}^{(\beta)} E_{i j} m_{j}^{(\beta)}$ similar to the procedure used in continuum crystal plasticity. Contours of the total slip $\Gamma \equiv \sum_{(\beta)}\left|\gamma^{(\beta)}\right|$ at $\bar{\varepsilon}_{22}=0.02$ are included in Figs. 5a and $5 \mathrm{~b}$ for a realization of the $h=0.25 \mu \mathrm{m}$ and $1 \mu \mathrm{m}$ unit cells, respectively. For the $h=0.25 \mu \mathrm{m}$ unit cell, deformation is mainly localised on slip planes that intersect the corners of the elastic particles while deformation is more spread in the larger $h=1 \mu \mathrm{m}$ unit cell. These differences in the deformation modes give rise to the size dependent plastic response as discussed subsequently.

The size dependence of the plastic part of the stress-strain response is usually associated with the higher density of geometrically necessary dislocations (GNDs) in the composites comprising a plastic matrix reinforced by elastic particles. This size dependence of the GND density is not borne out in Fig. 4b as we plot the total dislocation density rather than just the GND density. In DDP calculations, there is no definite way in which to differentiate between statistically stored dislocations (SSDs) and GNDs. The density of GNDs, $\rho_{\mathrm{GND}}$, is related to Nye's dislocation density tensor $\alpha_{i j}$ via $\alpha_{i j}=\sum_{(\beta)} \rho_{\mathrm{GND}} b^{(\beta)} s_{i}^{(\beta)} m_{j}^{(\beta)}$. However, $\rho_{\mathrm{GND}}$ cannot be uniquely extracted from this relation and hence here we choose to parameterise $\rho_{\mathrm{GND}}$ via the Euclidean norm $\|\alpha\| \equiv \sqrt{\alpha_{13}^{2}+\alpha_{23}^{2}}$ as described subsequently.

Under the assumption of infinitesimal deformations, Nye's tensor is related to the lattice curvature, viz. $\alpha_{i j}=-\omega_{j, i}$ where $\omega_{j}$ is lattice rotation. In the $2 \mathrm{D}$ plane strain model considered here, $\omega_{1}=\omega_{2}=0$ and $\omega_{3}=\left(\tilde{u}_{2,1}^{(\mathrm{m})}+\hat{u}_{2,1}^{(\mathrm{m})}-\tilde{u}_{1,2}^{(\mathrm{m})}-\right.$ $\left.\hat{u}_{1,2}^{(\mathrm{m})}\right) / 2$ where the spatial derivatives of the ( () are computed analytically in order to not include the slip contributions. Thus, the only non-zero components of the Nye's tensor are $\alpha_{13}$ and $\alpha_{23}$ which motivates the choice of the norm $\|\alpha\|$ defined above. Corresponding to the slip distributions in Figs. 5a and 5b, contours of the normalized norm $\|\alpha\| / b$ at $\bar{\varepsilon}_{22}=0.02$ are included in Figs. $5 \mathrm{c}$ and $5 \mathrm{~d}$. Comparing the distributions for unit cells of sizes $h=0.25 \mu \mathrm{m}$ and $1 \mu \mathrm{m}$ it 

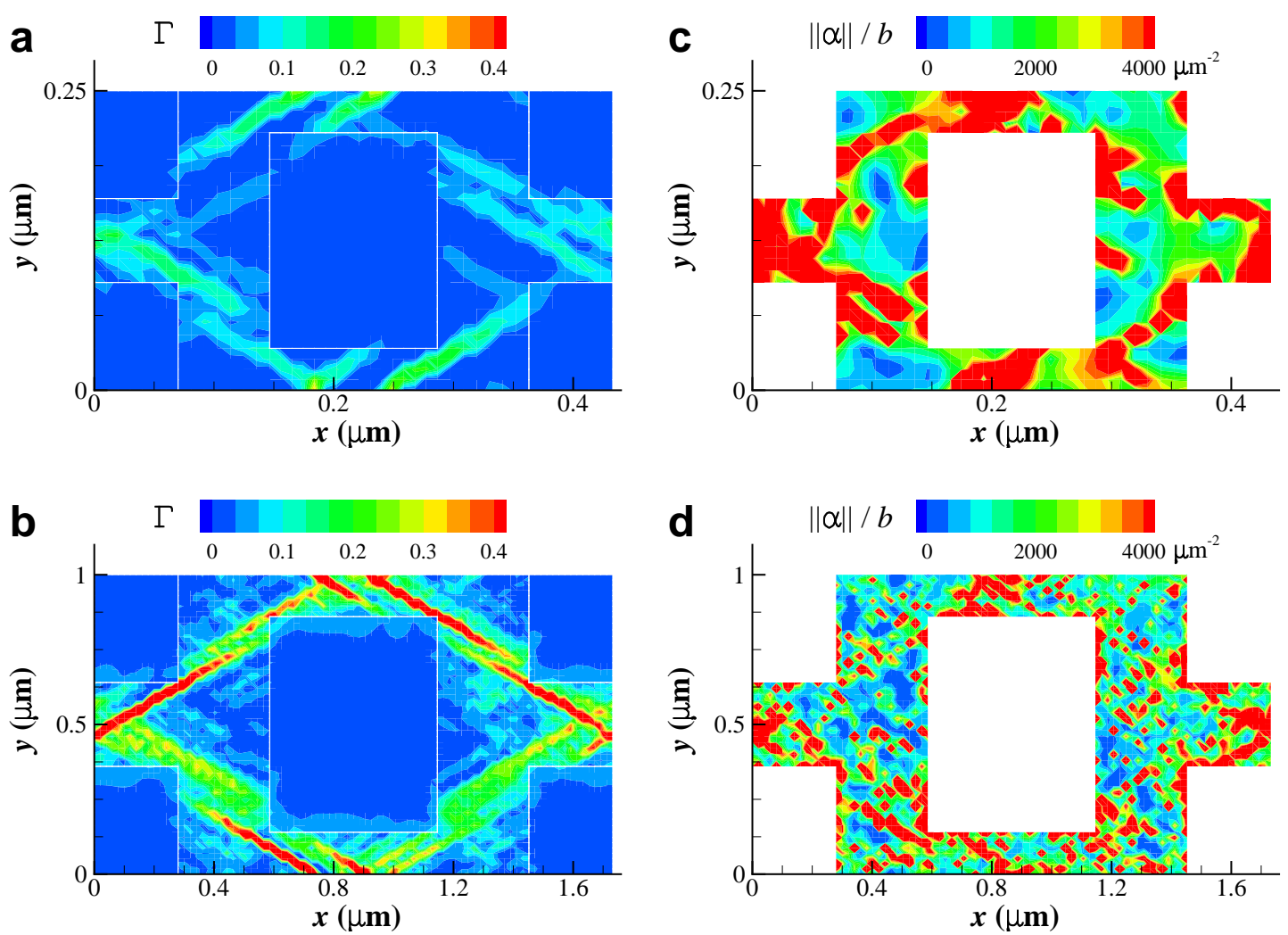

Figure 5: Distribution of $(\mathrm{a}, \mathrm{b})$ the total slip $\Gamma$ and $(\mathrm{c}, \mathrm{d})$ the normalized Euclidean norm of Nye's dislocation density tensor $\|\alpha\| / b$ at an applied strain $\bar{\varepsilon}_{22}=0.02$ in composites with glide-only motion of dislocations and unit cell sizes (a, c) $h=0.25 \mu \mathrm{m}$ and (b, d) $h=1 \mu \mathrm{m}$. The results are shown in the case where the interfacial diffusion constant $\mathscr{D}^{*}=0$.

is evident that the GND densities as parameterised by $\|\alpha\|$ are higher for the smaller unit cell ${ }^{2}$. This indicates that the approximate size independence of $\rho_{\text {dis }}$ is due to the fact that there is an increase in the density of SSDs with increasing size of the unit cell that compensates for the decrease in GND density. To further substantiate this we include in Figs. $6 \mathrm{a}$ and $6 \mathrm{~b}$ predictions of the corresponding dislocation structures at $\bar{\varepsilon}_{22}=0.02$ for unit cells of sizes $h=0.25 \mu \mathrm{m}$ and $1 \mu \mathrm{m}$, respectively. Large dislocation pile-ups are observed at the corners of the elastic particles in the $h=0.25 \mu \mathrm{m}$ unit cell (see Fig. 6a) and these pile-ups are of course associated with GNDs (see Fig. 5c). The dislocation density elsewhere within the matrix is low suggesting a low SSD density and consistent with the absence of slip in the bulk of the matrix (Fig. 5a). By contrast, there is high dislocation density everywhere within the matrix in the $h=1 \mu \mathrm{m}$ unit cell (see Fig. 6b): these dislocations carry slip as seen in the contours of $\Gamma$ in Fig. $5 b$ but mostly will not be classified as GNDs (parameterised by $\|\alpha\|$ ) as evident from Fig. $5 \mathrm{~d}$.

Now consider the case when interfacial diffusion is permitted with $\mathscr{D}^{*}=0.65$. The matrix/particle interface is now deformable due to deformation via interfacial diffusion. The interface thus supports smaller stresses and the dislocation pile-up lengths are reduced as can be seen by comparing the dislocation structures for the $h=0.25 \mu \mathrm{m}$

\footnotetext{
${ }^{2}$ The area averages for the $h=0.25 \mu \mathrm{m}$ and $1 \mu \mathrm{m}$ unit cells are $\sim 8300$ and $\sim 6000 \mu \mathrm{m}^{-2}$, respectively.
} 

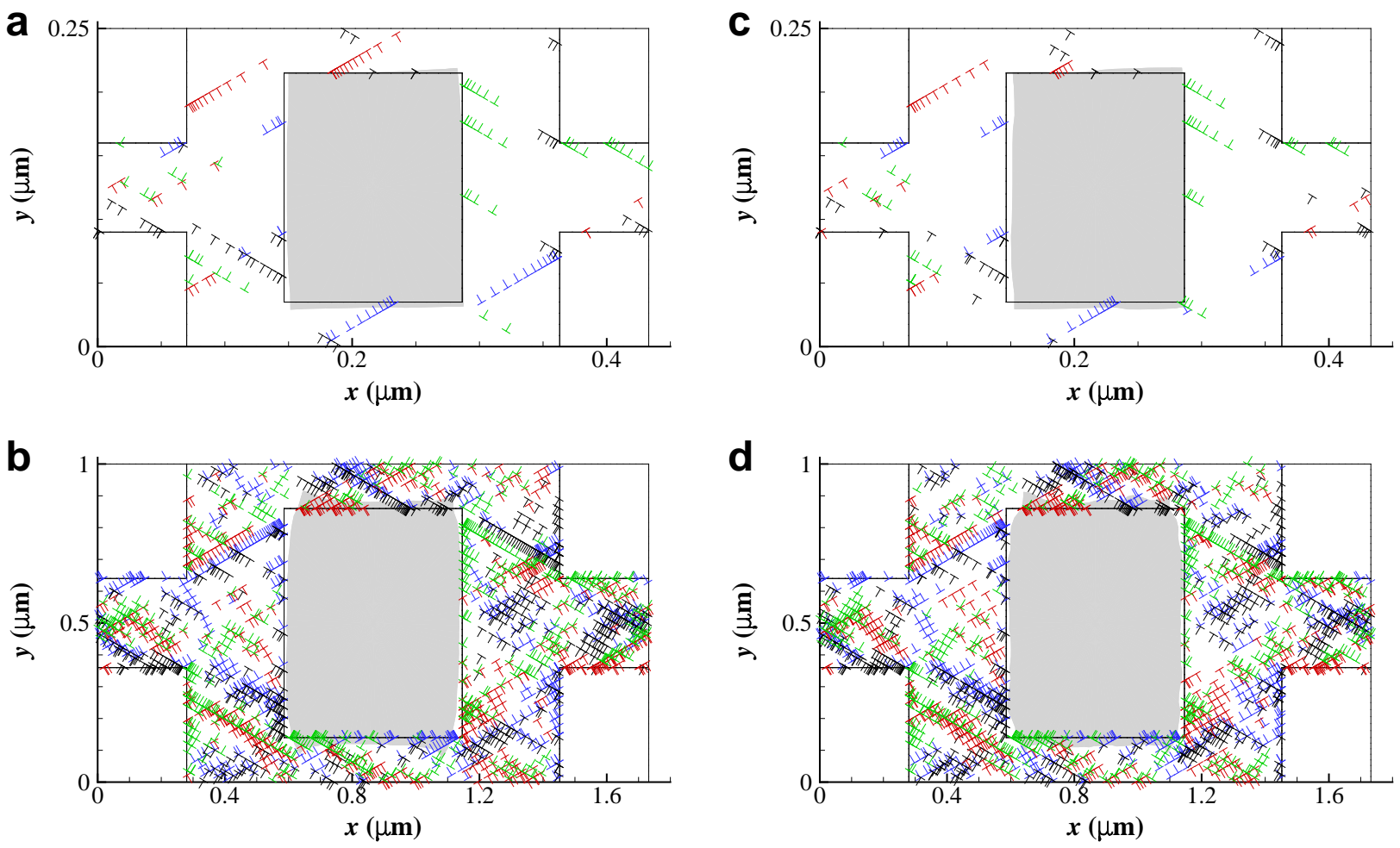

Figure 6: The dislocation distributions in the composites with glide-only motion of dislocations at an applied strain $\bar{\varepsilon}_{22}=0.02$. Results are shown for composites with (a) $h=0.25 \mu \mathrm{m}$ and (b) $h=1 \mu \mathrm{m}$ unit cells with no interfacial diffusion $\left(\mathscr{D}^{*}=0\right)$ as well as for (c) $h=0.25 \mu \mathrm{m}$ and (d) $h=1 \mu \mathrm{m}$ unit cells with an interfacial diffusion constant $\mathscr{D}^{*}=0.65$. The deformed shape of the central particle is also illustrated in grey in each case with displacements magnified by a factor of 2.5 in (a) and (c) and by a factor of 10 in (b) and (d). The colours assigned to dislocations are defined in Fig. 3.

unit cells for $\mathscr{D}^{*}=0$ and 0.65 in Figs. $6 \mathrm{a}$ and $6 \mathrm{c}$, respectively. As a consequence the plastic hardening rate is reduced especially for the smaller unit cells (Fig. 4a) even though the dislocation density is unaffected by the inclusion of interfacial diffusion as seen in Fig. 4b. Deformation of the particles now occurs by a combination of elastic and interfacial diffusional deformation. The deformed shapes of the central particles are illustrated in Fig. 6 where for the sake of clarity the deformations are magnified by a factor of 2.5 and 10 for $h=0.25 \mu \mathrm{m}$ and $h=1 \mu \mathrm{m}$ unit cells, respectively. In order to further quantify this deformation, we define two measures of average strain within the particle as follows. The rate of displacement of the surface of the particles due to purely elastic deformation is given by $\dot{u}_{i}^{(\mathrm{p})}$ and thus the volume average elastic strain within the particle follows as

$$
\bar{\varepsilon}_{i j}^{(\mathrm{p})-\mathrm{el}} \equiv \frac{1}{A^{(\mathrm{p})}} \int_{A^{(\mathrm{p})}} \varepsilon_{i j}^{(\mathrm{p})} d A=\frac{1}{2 A^{(\mathrm{p})}} \oint_{C^{(\mathrm{p})}}\left(u_{i}^{(\mathrm{p})} n_{j}^{(\mathrm{p})}+u_{j}^{(\mathrm{p})} n_{i}^{(\mathrm{p})}\right) \mathrm{d} C,
$$

where $A^{(\mathrm{p})}$ and $C^{(\mathrm{p})}$ are the particle area and perimeter, respectively while $\varepsilon_{i j}^{(\mathrm{p})}$ is the accumulated strain within the particle given by the time integral of $\dot{\varepsilon}_{i j}^{(\mathrm{p})}$. However, the total deformation of the particle is due to both elastic deformation 
and interfacial diffusion with the total displacement rate of the particle surface given via Eq. (5) as

$$
\dot{\mathscr{U}}^{(\mathrm{p})}=\dot{u}_{i}^{(\mathrm{p})}-\Delta v_{n} n_{i}^{(\mathrm{m})} .
$$

Then, analogous to Eq. (22) we define a total average strain of the particle as

$$
\bar{\varepsilon}_{i j}^{(\mathrm{p})}=\frac{1}{2 A^{(\mathrm{p})}} \oint_{C^{(\mathrm{p})}}\left(\mathscr{U}_{i}^{(\mathrm{p})} n_{j}^{(\mathrm{p})}+\mathscr{U}_{j}^{(\mathrm{p})} n_{i}^{(\mathrm{p})}\right) \mathrm{d} C,
$$

where $\mathscr{U}_{i}^{(\mathrm{p})}$ is the time integral of $\dot{\mathscr{U}}^{(\mathrm{p})}$. In the absence of interfacial diffusion $\left(\mathscr{D}^{*}=0\right)$ both these measures of average strain of course co-incide.

Predictions of the variations of $\bar{\varepsilon}_{22}^{(\mathrm{p})}$ and $\bar{\varepsilon}_{22}^{(\mathrm{p}) \text {-el }}$ with $\bar{\varepsilon}_{22}$ for the central particle of the unit cell are included in Figs. 7a and $7 \mathrm{~b}$ for $\mathscr{D}^{*}=0$ and $\mathscr{D}^{*}=0.65$, respectively. The results clearly show that the particles elongate in the direction of applied load (i.e. the $x_{2}$ direction) for both the $\mathscr{D}^{*}=0$ and 0.65 cases. The total elongation as parameterised by $\bar{\varepsilon}_{22}^{(\mathrm{p})}$ is higher in the presence of interfacial diffusion suggesting that the material within the particle flows from its sides to its top and bottom surfaces driven by the interfacial stress fields. This interfacial diffusion decreases with increasing unit cell size with $\bar{\varepsilon}_{22}^{(\mathrm{p})}$ and $\bar{\varepsilon}_{22}^{(\mathrm{p}) \text {-el }}$ nearly coinciding in the $h=2 \mu \mathrm{m}$ unit cell even when interfacial diffusion is present. This reduction in stress-driven interfacial diffusion is a manifestation of the predicted size effect with the stresses within the unit cell (and along the interfaces) decreasing with increasing $h$. This reduction in the stresses within the unit cell also results in a reduction in the elastic deformation of the particle with increasing $h$ (Fig. 7b). Thus, deformation is increasingly accommodated by plastic deformation of the matrix with increasing $h$ and consequently the hardening rate of the $\bar{\sigma}_{22}$ versus $\bar{\varepsilon}_{22}$ curves also decreases with increasing $h$ as seen in Fig. $4 \mathrm{a}$.
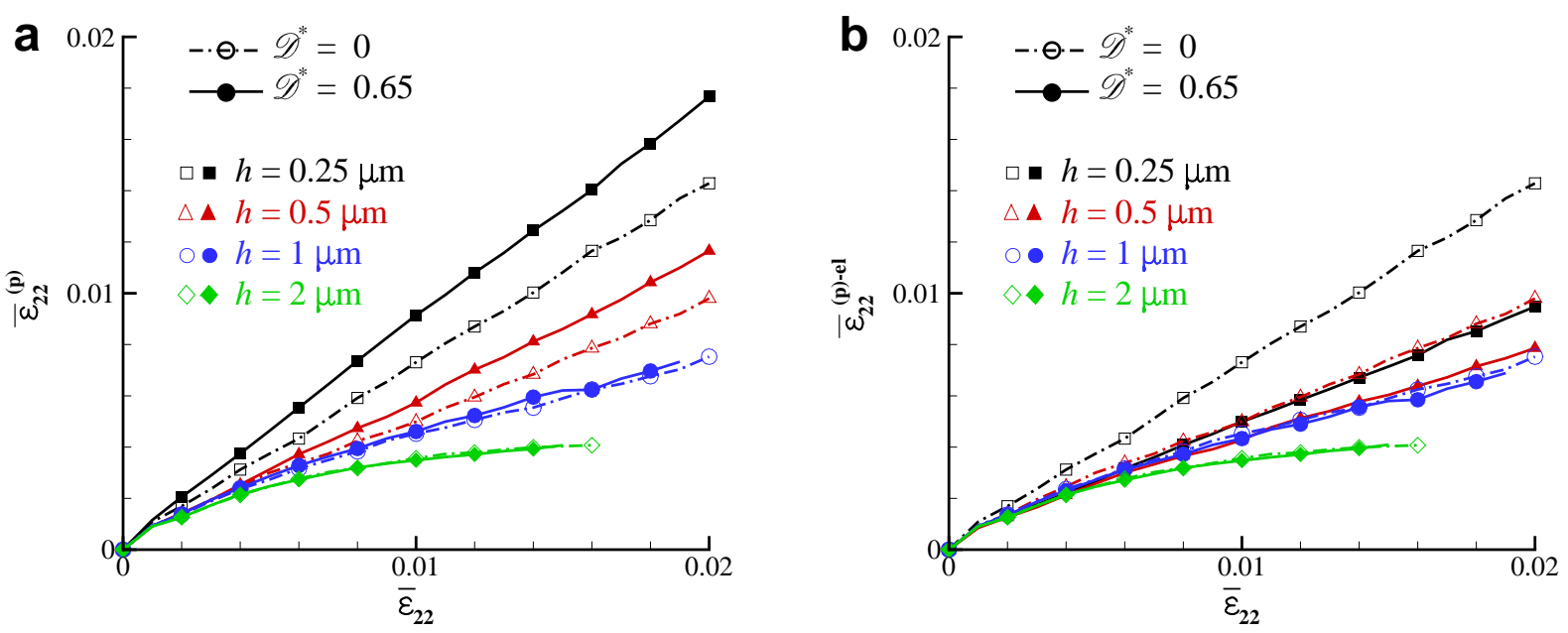

Figure 7: Predictions of the variation of the (a) total particle strain $\bar{\varepsilon}_{22}^{(\mathrm{p})}$ and (b) the elastic particle strain $\bar{\varepsilon}_{22}^{(\mathrm{p}) \text {-el }}$ with the applied strain $\bar{\varepsilon}_{22}$ for glide-only motion of dislocations. Results are shown for four values of unit cell sizes and the interfacial diffusion constant $\mathscr{D}^{*}=0$ and 0.65 . 


\subsection{Climb-assisted glide motion of dislocations}

Predictions of the tensile stress $\bar{\sigma}_{22}$ versus applied strain $\bar{\varepsilon}_{22}$ and the corresponding evolution of the dislocation density $\rho_{\text {dis }}$ with matrix deformation occurring by climb-assisted glide $\left(B_{\mathrm{c}} / B_{\mathrm{g}}=10^{3}\right)$ are included in Figs. $8 \mathrm{a}$ and $8 \mathrm{~b}$, respectively. Similar to the glide-only case, there is a clear size effect with the strength of the composite increasing with decreasing unit cell size and the strength in the presence of interfacial diffusion lower compared to the $\mathscr{D}^{*}=0$ case especially for the lower values of $h$. However, some key differences emerge compared to the glide-only case and these include:

(i) The stress versus strain response is no longer hardening even for the smallest $h=0.25 \mu \mathrm{m}$ unit cell. In fact the plastic responses are mildly softening in all cases.

(ii) The dislocation densities show a clear size effect with dislocation density higher for the smaller unit cells. Moreover, the dislocation density is affected by the presence of interfacial diffusion especially for the smaller unit cells: the dislocation density is higher for the $\mathscr{D}^{*}=0.65$ case compared to the $\mathscr{D}^{*}=0$ case.
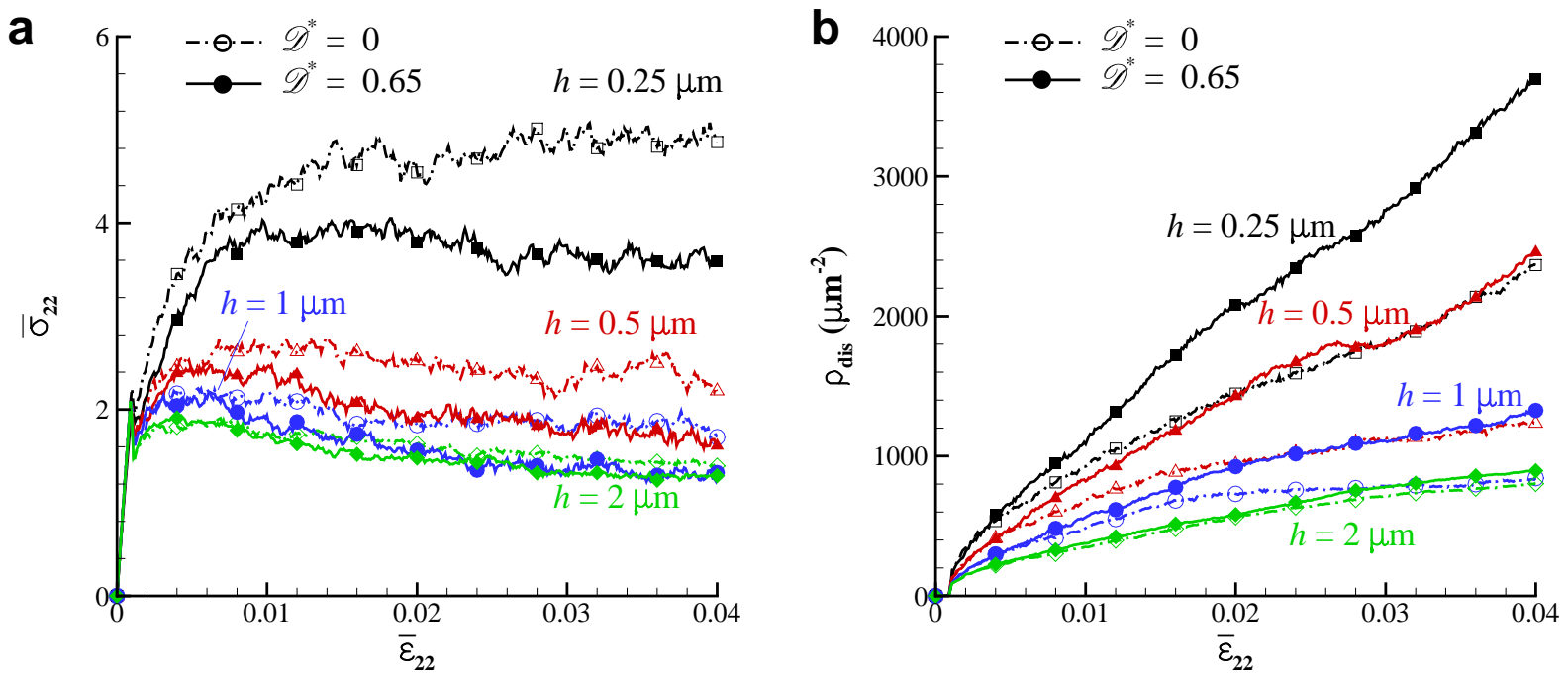

Figure 8: (a) The normalised tensile stress $\bar{\sigma}_{22}$ versus strain $\bar{\varepsilon}_{22}$ response of the composite with climb-assisted glide motion of dislocations $\left(B_{\mathrm{c}} / B_{\mathrm{g}}=10^{3}\right)$ and $(\mathrm{b})$ the corresponding evolution of dislocation density $\rho_{\mathrm{dis}}$ in the matrix. Results are shown for four unit cell sizes $h$ as well as for interfacial diffusion constants $\mathscr{D}^{*}=0$ and 0.65 .

We proceed to rationalise these differences by first restricting attention to the $\mathscr{D}^{*}=0$ case. The distribution of dislocation sources and obstacles is shown in Fig. 9a for a single realisation of the $h=1 \mu \mathrm{m}$ unit cell while Figs. 9b9f show the evolution of the dislocation structure for $\bar{\varepsilon}_{22} \leq 0.03$. Early in the deformation the dislocations are dispersed reasonably uniformly within the matrix but as the deformation proceeds, climb of the dislocations allows the dislocations to arrange themselves first into low energy wall structures (Figs. 9c and 9d). Further deformation results in the formation of dislocation cells with the cell size decreasing with increasing deformation. The prediction 

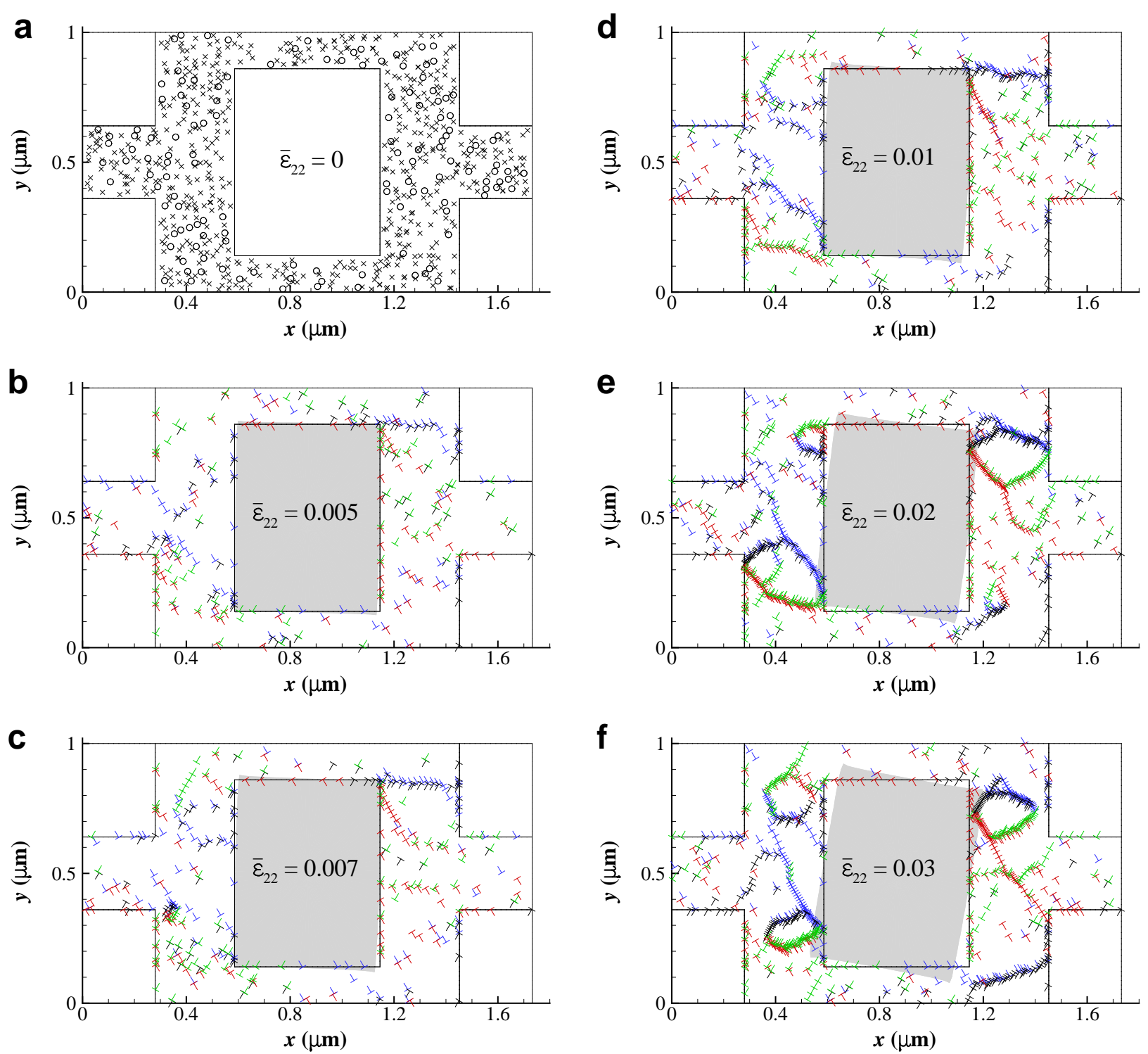

Figure 9: (a) Distribution of sources (circles) and obstacles (crosses) within the matrix of the composite with the $h=1 \mu \mathrm{m}$ unit cell. The evolution of the dislocation structure in this unit cell is shown in (b)-(f) for the climb-assisted glide case $\left(B_{\mathrm{c}} / B_{\mathrm{g}}=10^{3}\right)$ and interfacial diffusion constant $\mathscr{D}^{*}=0$. The deformed shape of central particle is indicated in grey in each case with displacements magnified by a factor of 10 and the value of strain $\bar{\varepsilon}_{22}$ is also indicated on the central particle. The colours assigned to dislocations are defined in Fig. 3.

of these cell structures is in line with observations reported for Ni superalloys; see for example Pollock and Argon (1992) and more recently Agudo Jacome et al. (2013, 2014). The cell walls provide pathways where dislocation climb is easier and we attribute the observed softening in the stress versus strain response to the refining of the dislocation cell structure. We emphasize that the dislocation cell structures are uncorrelated with the dislocation source and obstacle distributions shown in Fig. 9a. The dislocation structures at $\bar{\varepsilon}_{22}=0.04$ in unit cells ranging in size from $h=0.25 \mu \mathrm{m}$ to $h=2 \mu \mathrm{m}$ (a realization for each case) are plotted in Fig. 10a-d. Dislocation cells are observed to form 

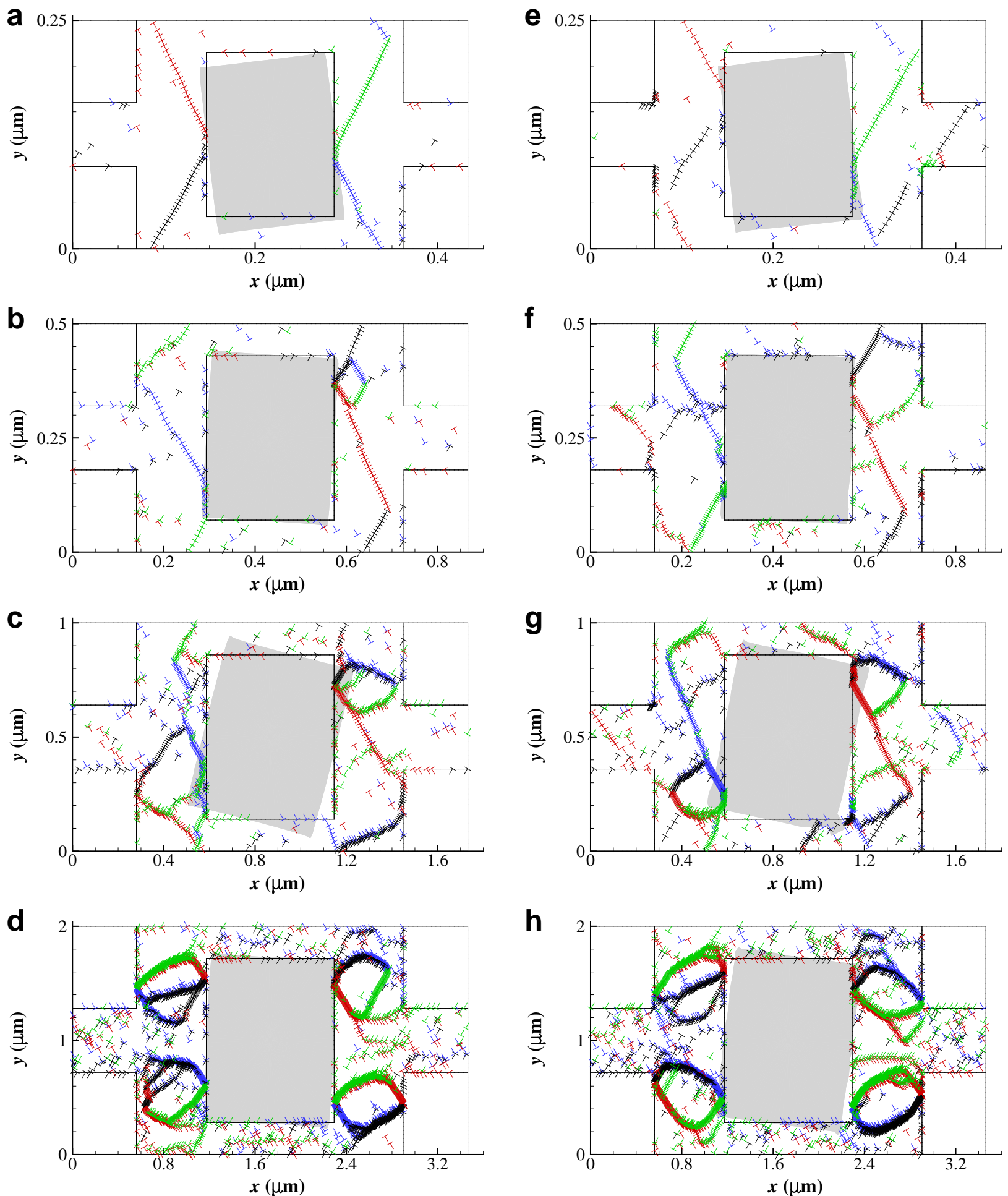

Figure 10: The dislocation distributions in composites with unit cell sizes in the range $0.25 \mu \mathrm{m} \leq h \leq 2 \mu \mathrm{m}$ at an applied strain $\bar{\varepsilon}_{22}=0.04$ with climb-assisted glide motion of the dislocations $\left(B_{\mathrm{c}} / B_{\mathrm{g}}=10^{3}\right)$. Results are shown in (a)-(d) for $\mathscr{D}^{*}=0$ and in (e)-(h) for $\mathscr{D}^{*}=0.65$. The deformed shape of the central particle is indicated in grey in each case with displacements magnified by a factor of 2.5 in (a) and (e), factor of 5 in (b) and (f), a factor of $10 \mathrm{in}(\mathrm{c})$ and (g) and $20 \mathrm{in}(\mathrm{d})$ and (h). The colours assigned to dislocations are defined in Fig. 3. 
in all cases and the majority of dislocations lie within these cell walls. Lattice curvatures are associated with these dislocation cell structures and in that sense we argue that the majority of dislocations in the matrix when dislocation motion is by climb-assisted glide are "geometrically necessary" with dislocation climb resulting in the annihilation of most of the SSDs. Thus, unlike in the glide-only case we expect the total dislocation density within the matrix to strongly correlate with the GND density. The GND density within the matrix is expected to increase with decreasing $h$ and this is reflected in the total dislocation densities plotted in Fig. 8b.

Now consider the case when interfacial diffusion is enabled with $\mathscr{D}^{*}=0.65$. Similar to the glide-only case, interfacial diffusion reduces the strength of the composite especially at small values of $h$ (Fig. 8a). In the glide only case this reduction in strength is due to larger particle deformation resulting from interfacial diffusion. In order to investigate whether this mechanism is still present when dislocation climb is enabled, we include in Fig. 11a predictions of the variation of $\bar{\varepsilon}_{22}^{(\mathrm{p})}$ (cf. Eq. (24)) with $\bar{\varepsilon}_{22}$ in this climb-assisted glide case for both $\mathscr{D}^{*}=0$ and 0.65. Surprisingly, the total elongation of the particles as parameterised by $\bar{\varepsilon}_{22}^{(\mathrm{p})}$ are independent of $\mathscr{D}^{*}$ (to within the variations between the different realisations of the source and obstacle distributions within the unit cells) though of course $\bar{\varepsilon}_{22}^{(\mathrm{p})}$ increases with decreasing $h$. Thus, unlike the glide-only case, we cannot attribute the weaker response of the composite when interfacial diffusion is active to additional deformation of the particle. It then follows that the weaker response of the composite must be due to interfacial diffusion permitting easier deformation of the matrix when dislocation climb is permitted. We proceed to clarify this change in deformation mechanism within the matrix.
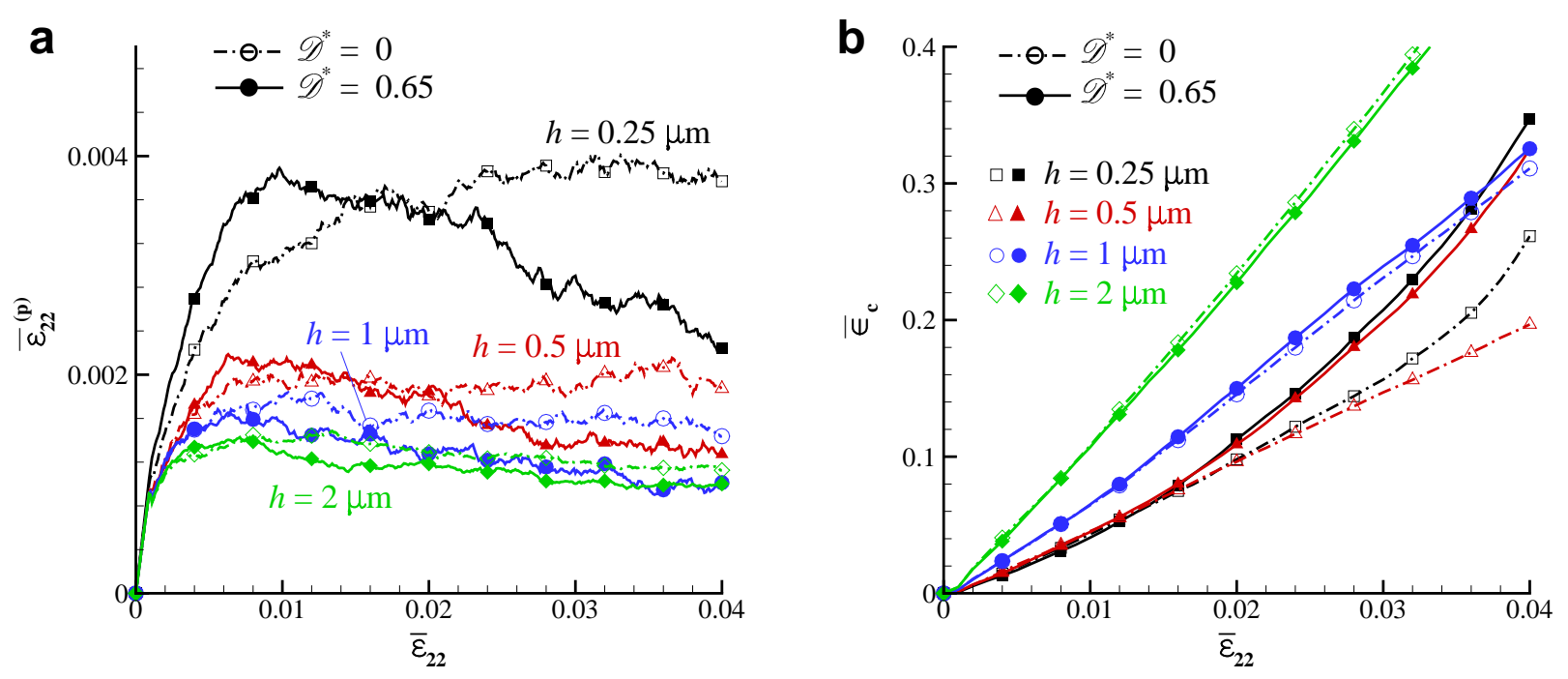

Figure 11: Predictions of the variation of the (a) total particle strain $\bar{\varepsilon}_{22}^{(\mathrm{p})}$ and (b) the average strain $\bar{\epsilon}_{\mathrm{c}}$ due to dislocation climb with the applied strain $\bar{\varepsilon}_{22}$ for climb-assisted glide motion of dislocations $\left(B_{\mathrm{c}} / B_{\mathrm{g}}=10^{3}\right)$. Results are shown for four values of unit cell sizes and the interfacial diffusion constants $\mathscr{D}^{*}=0$ and 0.65 .

The evolution of the dislocation density plotted in Fig. $8 \mathrm{~b}$ shows that $\rho_{\text {dis }}$ within the matrix is higher in the $\mathscr{D}^{*}=0.65$ case compared to when no interfacial diffusion is permitted with $\mathscr{D}^{*}=0$. With interfacial diffusion active, the matrix/particle interfaces are deformable. This reduces back stresses on dislocation sources within the relatively 
narrow matrix channels and permits easier nucleation of new dislocations as discussed in the context of the shear of single crystals sandwiched between deformable elastic layers by Danas et al. (2010). The higher nucleation rate then increases the overall dislocation density. The dislocation structures within the composite unit cell at $\bar{\varepsilon}_{22}=0.04$ are included in Figs. 10e-h for the $\mathscr{D}^{*}=0.65$ case and the four unit cell sizes investigated here. While the dislocation structures look reasonably similar to the $\mathscr{D}^{*}=0$ case with dislocation cells forming within the matrix, a closer inspection reveals that the higher overall dislocation density results in dislocations being more densely packed within the cell walls in the $\mathscr{D}^{*}=0.65$ case. Recall that dislocation climb is accelerated along these cell walls and hence we argue that interfacial diffusion enhances dislocation climb within the matrix and this results in a weaker response of the composite. To quantify this increase in dislocation climb within the matrix, we define a measure of average strain within the matrix due to dislocation climb as

$$
\bar{\epsilon}_{\mathrm{c}}=\frac{1}{A^{(\mathrm{m})}} \int_{A^{(\mathrm{m})}} \sum_{\beta=1}^{2}\left|m_{i}^{(\beta)} E_{i j} m_{j}^{(\beta)}\right| \mathrm{d} A,
$$

where $A^{(\mathrm{m})}$ is the area of the matrix and $E_{i j}$ the strain calculated by numerically differentiating the total displacement field $u_{i}$ using square bi-linear elements of size $0.01 \mu \mathrm{m}$ as detailed in Section 4.1. Predictions of the variation of $\bar{\epsilon}_{\mathrm{c}}$ with $\bar{\varepsilon}_{22}$ are plotted in Fig. $11 \mathrm{~b}$ for the four unit cell sizes considered here and $\mathscr{D}^{*}=0$ and 0.65 . It is clear that climb deformation is enhanced when interfacial diffusion is present, especially at the smaller values of $h$ consistent with the hypothesis presented above. We note in passing that consistent with the reduction in strength of the composite with increasing $h$ (Fig. 8a), the results in Fig. 11b confirm that this strength reduction is associated with increasing climb deformation within the matrix with increasing $h$.

We note in passing that the deformation of the central particle shown in Figs. 9 and 10 suggests that some of the matrix deformation is accommodated by rotation of the particles. In the simulations reported here, this rotation was typically $2^{\circ}$ independent of the unit cell size with rotations up to approximately $5^{\circ}$ in some of the realisations. The statistical nature of the distribution of sources and obstacles within the matrix implies that the sense of the rotation is random for the imposed uniaxial tensile loading with approximately equal number of realisations having central particles that rotate clockwise and counter-clockwise.

\subsubsection{Effect of the climb rate of dislocations $B_{\mathrm{c}} / B_{\mathrm{g}}$}

The results reported earlier demonstrate the large influence of dislocation climb on the response of the composites: while a strain hardening stress versus strain response was predicted in the glide-only limit (cf. Fig. 4a), the stress versus strain response displayed a distinct peak stress when dislocation climb was permitted with $B_{\mathrm{c}} / B_{\mathrm{g}}=10^{3}$ (cf. Fig. 8a). The softening in the presence of dislocation climb was related to the formation of dislocation cell structures within the matrix and consequent softening of the matrix. To investigate the effect of the dislocation climb rate, we include in Fig. 12 predictions of the tensile responses of the composite with $B_{\mathrm{c}} / B_{\mathrm{g}}=10^{5}$ for both $\mathscr{D}^{*}=0$ and 0.65 and the four unit cell sizes considered here. While the qualitative responses are similar to the $B_{\mathrm{c}} / B_{\mathrm{g}}=10^{3}$ case in Fig. $8 \mathrm{a}$, two key differences are noted: (i) the effect of interfacial diffusion is mild and (ii) the peak stress is reached at the 


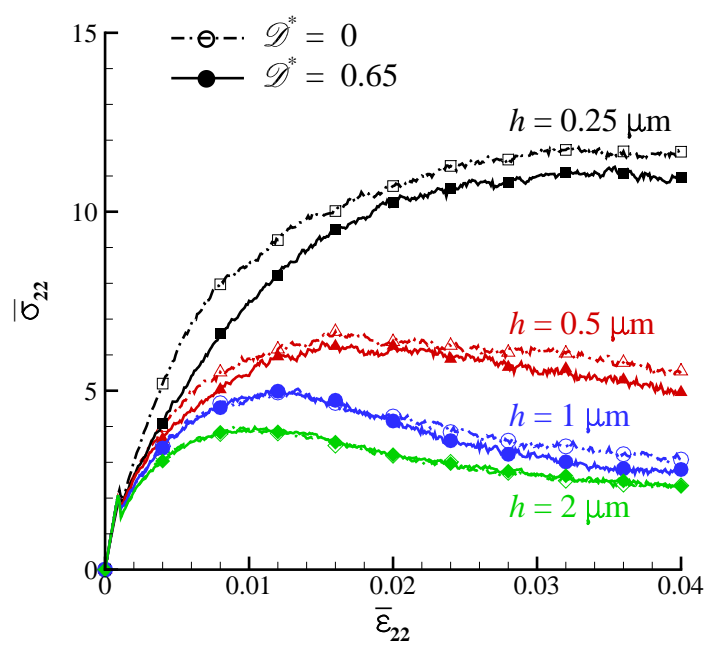

Figure 12: The normalised tensile stress $\bar{\sigma}_{22}$ versus strain $\bar{\varepsilon}_{22}$ response of the composite with climb-assisted glide motion of dislocations $\left(B_{\mathrm{c}} / B_{\mathrm{g}}=\right.$ $10^{5}$ ). Results are shown for four unit cell sizes $h$ as well as for interfacial diffusion constants $\mathscr{D}^{*}=0$ and 0.65.

higher value of the applied strain. These differences are rationalised by recalling that interfacial diffusion has a more significant effect on the climb of dislocations and thus reducing the climb rate also reduces the effect of interfacial diffusion. Moreover, the climb rate of dislocations sets the time-scale over which the dislocations can arrange into cell structures that soften the matrix. This implies that for a fixed applied strain rate, the applied strain at which the matrix begins to soften appreciably increases with decreasing climb rate (or increasing $B_{\mathrm{c}} / B_{\mathrm{g}}$ ) as confirmed by comparing Figs. 8a and 12.

\subsubsection{Effect of the interfacial diffusion constant $\mathscr{D}^{*}$}

The results presented above for the $\mathscr{D}^{*}=0$ and 0.65 cases demonstrate that interfacial diffusion reduces the strength of the composite but in the presence of climb-assisted glide motion of the dislocations, the deformation of the particles is reasonably unaffected by interfacial diffusion. Here we check the generality of this rather surprising finding by reporting predictions for $\mathscr{D}^{*}$ over the range $0 \leq \mathscr{D}^{*} \leq 6.5$. For the sake of brevity we restrict ourselves here to the $h=1 \mu \mathrm{m}$ unit cell; the qualitative conclusions remain unchanged for all four unit cell sizes considered here.

Predictions of the variation of normalized stress $\bar{\sigma}_{22}$, dislocation density $\rho_{\text {dis }}$ and average particle strain $\bar{\varepsilon}_{22}^{(\mathrm{p})}$ with applied strain $\bar{\varepsilon}_{22}$ are included in Figs. $13 \mathrm{a}, \mathrm{b}$ and c, respectively for five selected values of $\mathscr{D}^{*}$. The stress versus strain response until the peak stress is reasonably unaffected by $\mathscr{D}^{*}$ but beyond the peak stress the softening rate of the composite increases with increasing $\mathscr{D}^{*}$. Correspondingly the $\rho_{\text {dis }}$ and $\bar{\varepsilon}_{22}^{(\mathrm{p})}$ variations early in the deformation are also independent of $\mathscr{D}^{*}$. However, at higher applied strains the dislocation density is higher for the larger values of $\mathscr{D}^{*}$. By contrast, to within the variations between different unit cell realisations in the DDP calculations, $\bar{\varepsilon}_{22}^{(\mathrm{p})}$ is independent of $\mathscr{D}^{*}$. This confirms the overall conclusion that the enhanced softening associated with increasing $\mathscr{D}^{*}$ is a result of increased softening of the matrix in the presence of interfacial diffusion rather than additional deformation of the particles. 

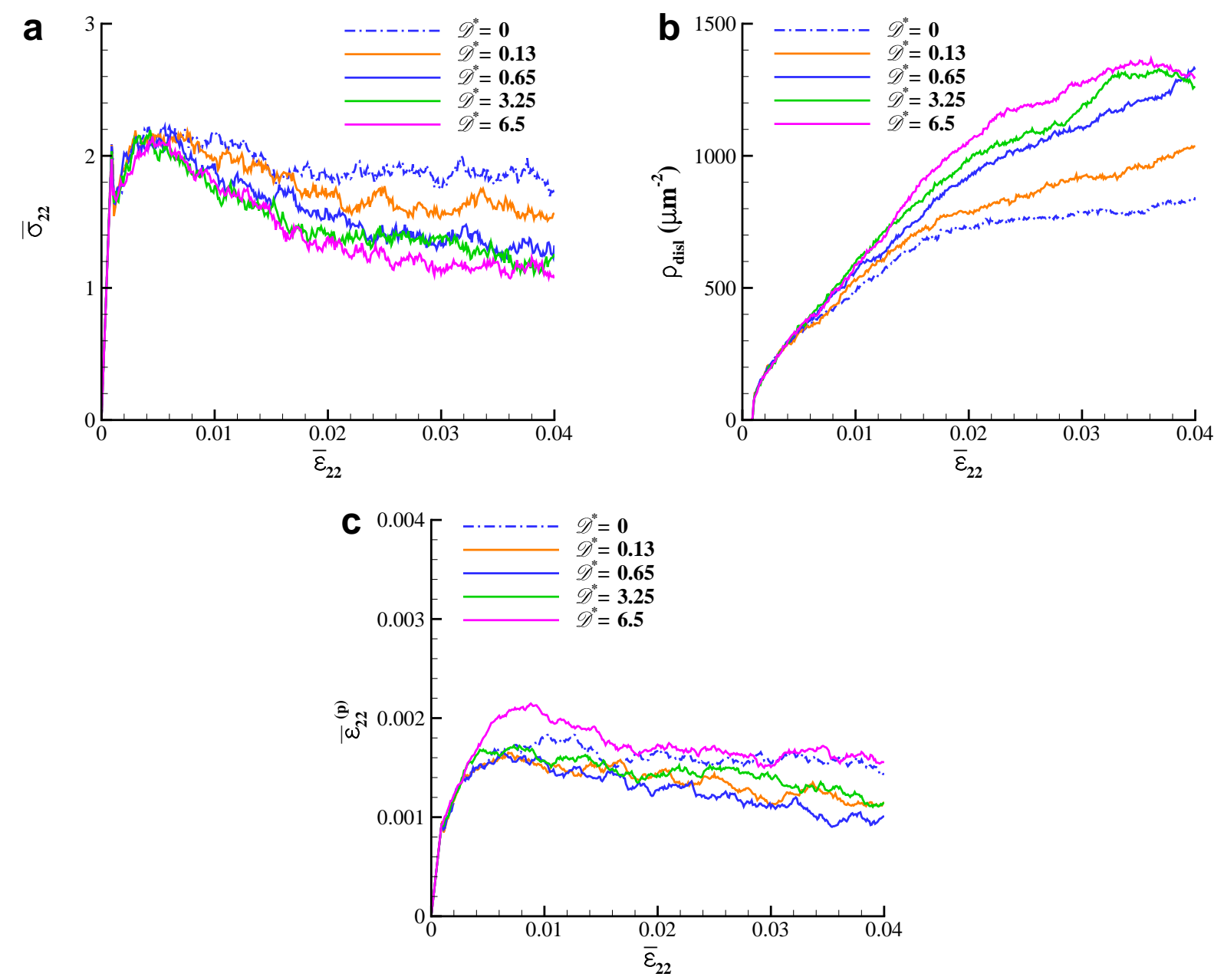

Figure 13: Predictions of variation of the (a) normalised tensile stress $\bar{\sigma}_{22}$ (b) dislocation density $\rho_{\text {dis }}$ in the matrix and (c) total particle strain $\bar{\varepsilon}_{22}^{(\text {p) }}$ with applied $\bar{\varepsilon}_{22}$ in the composite with the $h=1 \mu \mathrm{m}$ unit cell and climb-assisted glide motion of dislocations $\left(B_{\mathrm{c}} / B_{\mathrm{g}}=10^{3}\right)$. Results are shown for five interfacial diffusion constants in the range $0 \leq \mathscr{D}^{*} \leq 6.5$.

\subsection{Summary of the influence of unit cell size on the composite strength}

A summary of the flow strength of the composites as a function of the unit cell size $h$ is presented in Fig. 14a. The flow strength $\left\langle\bar{\sigma}_{22}\right\rangle$ is defined as the average value of $\bar{\sigma}_{22}$ over the range $0.015 \leq \bar{\varepsilon}_{22} \leq 0.02$ and results are presented in Fig. 14a for the two standard values of $\mathscr{D}^{*}=0$ and 0.65 . Furthermore, results are presented for $B_{\mathrm{c}} / B_{\mathrm{g}}=10^{4}$ and $10^{5}$ in addition to the reference values of $B_{\mathrm{c}} / B_{\mathrm{g}}=\infty$ (glide-only) and $B_{\mathrm{c}} / B_{\mathrm{g}}=10^{3}$ which is the reference value used for the climb-assisted glide calculations in the bulk of this study. In all cases, the flow strength decreases with increasing $h$ though this size dependence decreases as the climb rate increases with decreasing $B_{\mathrm{c}} / B_{\mathrm{g}}$. This effect is directly related to the lower energy dislocation cell structures that form and relax stresses in the matrix when dislocation climb is enabled as discussed above.

A superficial examination of Fig. 14a suggests that interfacial diffusion only has a significant effect on the flow 

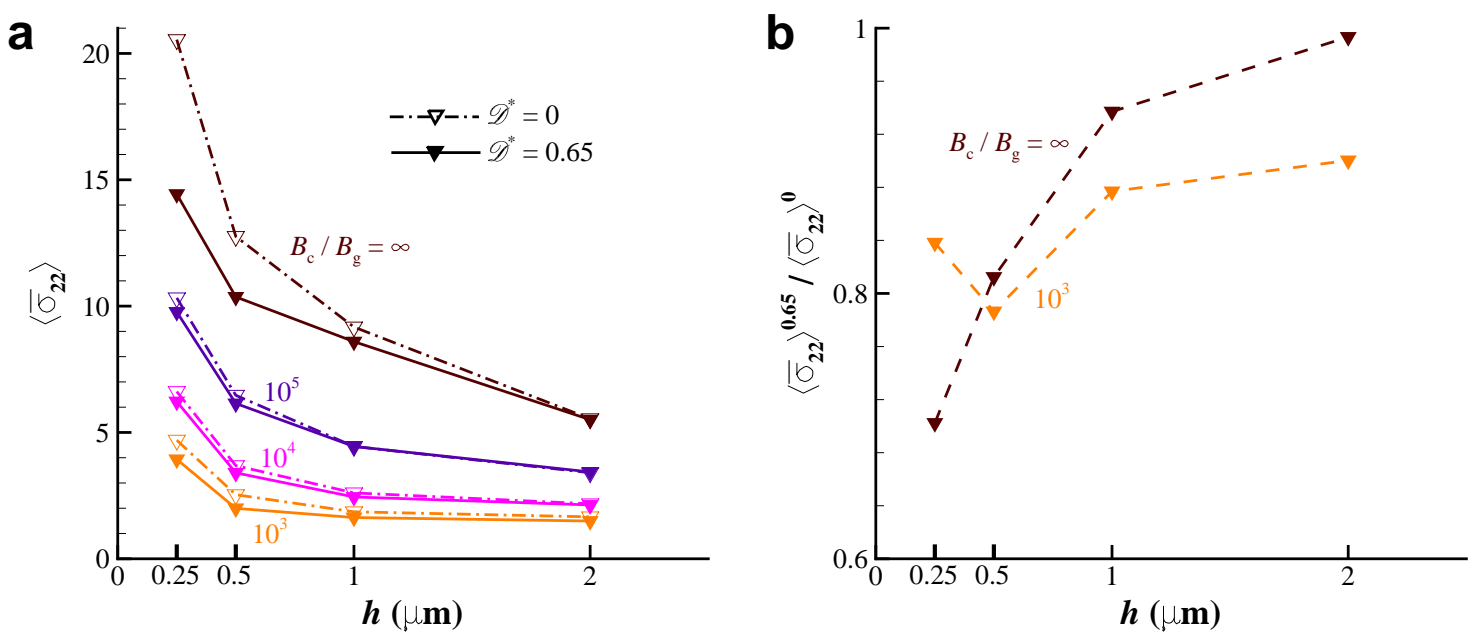

Figure 14: (a) The DDP predictions of the flow strength $\left\langle\bar{\sigma}_{22}\right\rangle$ of the composite as a function of the unit cell size $h$. Results are shown for four values of the climb drag co-efficient as parameterised by $B_{\mathrm{c}} / B_{\mathrm{g}}$. In each of these four cases predictions without interfacial diffusion $\left(\mathscr{D}^{*}=0\right)$ and an interfacial diffusion constant $\mathscr{D}^{*}=0.65$ are included. (b) The ratio of the flow strength with $\mathscr{D}^{*}=0.65$ and $\mathscr{D}^{*}=0\left(\left\langle\bar{\sigma}_{22}\right\rangle^{0.65} /\left\langle\bar{\sigma}_{22}\right\rangle^{0}\right)$ for $B_{\mathrm{c}} / B_{\mathrm{g}}=\infty$ and $10^{3}$ as a function of the unit cell size $h$.

strength at small values of $h$ when plasticity in the matrix is dominated by the glide motion of the dislocations. This is characteristic of the deformation that occurs when high volume fractions of coherent reinforcements are present (such as in the technologically important nickel-based superalloys). In these classes of materials dislocations glide with relative ease until they encounter precipitate interfaces. Local climb along the interfaces, rather than climb in the bulk matrix material, then dominates the process (Pollock and Argon, 1992). To better quantify the important role of interfacial diffusion we define $\left\langle\bar{\sigma}_{22}\right\rangle^{0.65} /\left\langle\bar{\sigma}_{22}\right\rangle^{0}$ which is the ratio of the flow strength with $\mathscr{D}^{*}=0.65$ and $\mathscr{D}^{*}=0$. This ratio is plotted in Fig. $14 \mathrm{~b}$ for $B_{\mathrm{c}} / B_{\mathrm{g}}=\infty$ and $B_{\mathrm{c}} / B_{\mathrm{g}}=10^{3}$. The effect of interfacial diffusion in reducing the flow strength increases with decreasing $h$ as diffusion needs to occur over small length scales to affect the same level of deformation. Moreover, the magnitude of the effect of interfacial diffusion is approximately the same in both the $B_{\mathrm{c}} / B_{\mathrm{g}}=\infty$ and $10^{3}$ cases. This is because even though the global stresses are much lower in the $B_{\mathrm{c}} / B_{\mathrm{g}}=10^{3}$, interfacial diffusion is driven by local dislocation stresses that are of similar level in both the glide-only and climb-assisted glide cases.

\subsection{Discussion}

The DDP framework presented here has been developed to model composites and/or two phase materials containing embedded "hard" coherent precipitates wherein the deformation of the matrix is by the climb-assisted glide motion of dislocation while deformation of second phase particles by a combination of elasticity and interfacial diffusion. This framework has been used to investigate the uniaxial tensile response of composite materials at high temperatures. In the current study while deformation of the particles via interfacial diffusion is explicitly modelled, vacancy diffusion resulting in dislocation climb within the matrix is not explicitly modelled. Rather the climb motion 
of dislocations is modelled via a drag relation similar to the glide motion expect that the climb motion of dislocation is significantly slower. This simplified representation of the climb motion as developed by Danas and Deshpande (2013) was employed mainly to reduce the computational cost of the simulations. A fully coupled model for vacancy diffusion and dislocation climb was developed by Ayas et al. (2014). In that study the authors demonstrated that while the simpler formulation of Danas and Deshpande (2013) slightly over-predicts the effect of dislocation climb, the results are qualitatively very similar. Thus given that the focus of the present study was to investigate the effect of interfacial diffusion, we employed the formulation of Danas and Deshpande (2013) for dislocation climb in order to reduce computational costs.

The 2D DDP calculations presented here are aimed at simulating high temperature deformation of composites wherein several three dimensional (3D) dislocation related phenomena may be important as well. For example, under room temperature conditions dislocation phenomena such as cross-slip and jog formation are known to influence plastic flow and significant advances have been made in 3D DDP simulations (see for example Arsenlis et al. (2007)) to capture these effects. However, these calculations remain computationally very expensive and complex boundary value problems such as the one analysed here remain intractable to 3D DDP. In order to circumvent this numerical issue, so-called 2.5D DDP techniques have been developed (Benzerga et al., 2004) for room temperature dislocation plasticity to approximately account for jog formation and the consequent creation of new Frank-Read sources. In high temperature DDP, additional 3D effects such as jog formation resulting in circumventing of obstacles are also now operative and it remains to quantify their role in affecting the material response. We propose that compared to full 3D DDP simulations, a more practical solution might be the extension of the 2.5D DDP formulations to high temperature deformations in order to include 3D effects.

Furthermore, we note that for the sake of brevity, parametric studies are reported here only for the effect of dislocation climb and interfacial diffusion. The other parameters have been selected so as to give results representative of Ni superalloys. For example, depending on the level of dislocation climb (which in turn is set by temperature), the strengths predicted here are in the realistic range $(2 \sim 20) \bar{\tau}_{\text {nuc }}=100 \sim 1000 \mathrm{MPa}$. However, it is worth emphasising here that calculations performed as part of this study (but not reported here) indicated that the results are insensitive to the obstacle density and display only a mild sensitivity to the source density over the range $300<\rho_{\text {nuc }}<$ $500 \mu \mathrm{m}^{-2}$. For higher source densities there is a decrease in the predicted strengths though the predictions remain qualitatively unchanged. Finally, we note that the simulations here render the reinforcements immune to shearing by dislocations. In systems with ceramic or intermetallic reinforcements, this is a realistic assumption. However, an interesting extension of the model would be to allow the dislocations to penetrate and glide across the reinforcements, to assess the role of precipitate shearing that, for example, occurs at high stresses in nickel-based superalloys.

The simulations presented here demonstrate, for the first time, a potentially important role for interfacial diffusion that arises only due to the presence of high volume fractions of small size reinforcements. Given that chemical gradients and segregation may also occur at such interfaces, the results point to new strategies for improving the high temperature strength of a wide range of materials that are strengthened by high volume fractions of coherent 
precipitates.

\section{Concluding remarks}

The high temperature discrete dislocation plasticity (DDP) framework has been extended to incorporate deformation of phases within the material via stress-driven interfacial diffusion. The deformation due to interfacial diffusion is modelled as a velocity discontinuity across the interface of the phases with this velocity dependent on the second spatial gradient of the stress along the interface. The calculation of these stress gradients is problematic in the DDP setting due to the singularities associated with the individual dislocations and the DDP superposition framework of Van der Giessen and Needleman (1995) is modified to enable the accurate calculation of the interfacial diffusion rates.

This modified DDP framework is employed to investigate the uniaxial tensile response of composites comprising a matrix where deformation occurs by a combination of glide and climb of dislocations and elastic particles which deform by a combination of elastic deformation and interfacial diffusion. The results are best summarised by dividing them into two cases: (i) when matrix deformation occurs only by the glide motion of dislocations and (ii) when dislocation motion is by climb-assisted glide. First consider the glide-only case. The strength of the composite is size dependent with the strength increasing with decreasing unit cell size even though the total dislocation density is size independent. The strength increases with decreasing unit cell size as back stresses due to dislocation pile-ups on matrix/particle interfaces have larger influences in the smaller unit cells with smaller matrix channels. However, the dislocation density is independent of unit cell size as the density of statistically stored dislocations (SSDs) increases with increasing unit cell size, compensating for the reduction in the density of geometrically necessary dislocations (GNDs). Deformation of the particles by interfacial diffusion increases the elongation of the particles in the direction of loading, consequently decreasing the strength of the composites when interfacial diffusion is active.

When deformation of the matrix is by climb-assisted glide, the strength of the composites is reduced as dislocations arrange themselves into lower energy cell structures rather than forming pile-ups at the matrix/particle interfaces. Another consequence of dislocation climb is that the SSDs largely annihilate. Thus, with GNDs dominating the dislocations within the matrix both the strength and total dislocation density increase with decreasing cell size. When interfacial diffusion is permitted in this case, the strength of the composite decreases but rather surprisingly interfacial diffusion does not result in an enhanced elongation of the particles. The decrease in the strength of the composites due to interfacial diffusion is a result of a change in the dislocation structures within the matrix that leads to a reduction in the matrix strength. Thus, the effect of interfacial diffusion in reducing strength is rather indirect in this case contrary to previous predictions that have employed continuum plasticity.

This preliminary investigation has demonstrated the potential of the climb-assisted glide DDP framework to investigate deformation mechanisms in multi-phase materials at high temperatures and especially the effect of interfacial diffusion. The applications of this framework to more complex problems such as the creep of nickel-based superalloys are topics for future studies. 


\section{Acknowledgments}

Support from ONR under grant number N62909-14-1N242 on Multi-scale methods for creep resistant alloys (program manager Dr. David Shifler) is gratefully acknowledged.

\section{References}

Agudo Jacome, L., Nortershauser, P., Heyer, J.-K., Lahni, A., Frenzel, J., Dlouhy, A., Somsen, C., Eggeler, G., 2013. High-temperature and low-stress creep anisotropy of single-crystal superalloys. Acta Mater 61, 2926-2943.

Agudo Jacome, L., Nortershauser, P., Somsen, C., Dlouhy, A., Eggeler, G., 2014. On the nature of phase cutting and its effect on high temperature and low stress creep anisotropy of ni-base single crystal superalloys. Acta Mater 69, 246-264.

Arsenlis, A., Cai, W., Tang, M., Rhee, M., Oppelstrup, T., Hommes, M. H. G., Pierce, T. G., Bulatov, V. V., 2007. Enabling strain hardening simulations with dislocation dynamics. Modell Simul Mater Sci Eng 15, 553-595.

Ayas, C., Dautzenberg, L. C. P., Geers, M. G. D., Deshpande, V. S., 2015. Climb-enabled discrete dislocation plasticity analysis of the deformation of a particle reinforced composte. J Appl Mech 82, 071007-1-13.

Ayas, C., van Dommelen, J. A. W., Deshpande, V. S., 2014. Climb-enabled discrete dislocation plasticity. J Mech Phys Solids 62, 113-136.

Benzerga, A. A., Brechet, Y., Needleman, A., Van der Giessen, E., 2004. Incorporating three-dimensional mechanisms into two-dimensional dislocation dynamics. Modell Simul Mater Sci Eng 12, 159-196.

Chakravarthy, S. S., Curtin, W. A., 2011. New algorithms for discrete dislocation modeling of fracture. Modell Simul Mater Sci Eng 19, 045009(12pp).

Cleveringa, H. H. M., Van der Giessen, E., Needleman, A., 1997. Comparison of discrete dislocation and continuum plasticity predictions for a composite material. Acta Mater 45, 3163-3179.

Cleveringa, H. H. M., Van der Giessen, E., Needleman, A., 1999. A discrete dislocation analysis of bending. Int J Plast 15, 837-868.

Danas, K., Deshpande, V. S., 2013. Plane-strain discrete dislocation plasticity with climb-assisted glide motion of dislocations. Modell Simul Mater Sci Eng 21, 045008(26pp).

Danas, K., Deshpande, V. S., Fleck, N. A., 2010. Compliant interfaces: A mechanism for relaxation of dislocation pile-ups in a sheared single crystal. Int J Plast 26, 1792-1805.

Geers, M. G. D., Cottura, M., Appolaire, B., Busso, E. P., Forest, S., Villani, A., 2014. Coupled glide-climb diffusion-enhanced crystal plasticity. J Mech Phys Solids 70, 136-153.

Haghighat, S. M. H., Eggeler, G., Raabe, D., 2013. Effect of climb on dislocation mechanisms and creep rates in -strengthened ni base superalloy single crystals: A discrete dislocation dynamics study. Acta Mater 61.

Hussein, M. I., Borg, U., Niordson, C. F., Deshpande, V. S., 2008. Plasticity size effects in voided crystals. J Mech Phys Solids 56, 114-131.

Ikeda, T., Almazouzi, A., Numakura, H., Koiwa, M., Sprengel, W., Nakajima, H., 1998. Single-phase interdiffusion in Ni 3 Al. Acta Metall Mater 46, 5369-5376.

Kamat, S. V., Hirth, J. P., Mehrabian, R., 1989. Mechanical properties of particulate-reinforced aluminum-matrix composites. Acta Metall 37, 2395-2402.

Keralavarma, S. M., Benzerga, A. A., 2015. High-temperature discrete dislocation plasticity. J Mech Phys Solids 82, 1-22.

Kubin, L. P., Canova, G., Condat, M., Devincre, B., Pontikis, V., Brechet, Y., 1992. Dislocation microstructures and plastic flow: a 3D simulation. Solid State Phenomena 23/24, 455-472.

Li, Y., Li, S., Li, Z., 2014. Interface diffusion-induced creep and stress relaxation in unidirectional metal matrix composites under biaxial loading. Mech Mater 76, 20-26.

Needleman, A., Rice, J. R., 1980. Plastic creep flow effects in the diffusive cavitation of grain boundaries. Acta Mettal 28, 1315-1332.

O’Day, M. P., Curtin, W. A., 2004. A superposition famework for discrete dislocation plastcity. J Appl Mech 71, 805-815. 
Pollock, T. M., Argon, A. S., 1992. Creep resistance of CMSX-3 nickel base superalloy single crystals. Acta Metall Mater 40, 1-30.

Rosler, J., Bao, G., Evans, A. G., 1991. The effects of diffusional relaxation on the creep strength of composites. Acta Mettal 39, $2733-2738$.

Segurado, J., LLorca, J., Romero, I., 2007. Computational issues in the simulation of two-dimensional discrete dislocation mechanics. Modell Simul Mater Sci Eng 15, S361-S375.

Shishvan, S. S., Mohammadi, S., Rahimian, M., Van der Giessen, E., 2011. Plane-strain discrete dislocation plasticity incorporating anisotropic elasticity. Int J Solids Struc 48, 374-387.

Sofronis, P., McMeeking, R. M., 1994. The effect of interface diffusion and slip on the creep resistance of particulate composite materials. Mech Mater 18, 55-68.

Van der Giessen, E., Needleman, A., 1995. Discrete dislocation plasticity: a simple planar model. Modell Simul Mater Sci Eng 3, 689-735.

Wang, X., Pan, E., 2011. Interaction between an edge dislocation and a circular inclusion with interface slip and diffusion. Acta Mater 59, 797-804.

Zhu, Z., Basoalto, H., Warnken, N., Reed, R. C., 2012. A model for the creep deformation behaviour of nickel-based single crystal superalloys. Acta Mater 60, 4888-4900. 